\title{
Free field primaries in general dimensions: counting and construction with rings and modules
}

\author{
Robert de Mello $\operatorname{Koch}^{a, b}$ and Sanjaye Ramgoolam ${ }^{b, c}$ \\ ${ }^{a}$ School of Physics and Telecommunication Engineering, South China Normal University, \\ Guangzhou 510006, China \\ ${ }^{b}$ National Institute for Theoretical Physics, School of Physics and Mandelstam Institute for \\ Theoretical Physics, University of the Witwatersrand, \\ Wits, 2050, South Africa \\ ${ }^{c}$ Centre for Research in String Theory, School of Physics and Astronomy, \\ Queen Mary University of London, \\ Mile End Road, London E1 4NS, U.K. \\ E-mail: robert@neo.phys.wits.ac.za, s.ramgoolam@quul.ac.uk
}

ABSTRACT: We define lowest weight polynomials (LWPs), motivated by $s o(d, 2)$ representation theory, as elements of the polynomial ring over $d \times n$ variables obeying a system of first and second order partial differential equations. LWPs invariant under $S_{n}$ correspond to primary fields in free scalar field theory in $d$ dimensions, constructed from $n$ fields. The LWPs are in one-to-one correspondence with a quotient of the polynomial ring in $d \times(n-1)$ variables by an ideal generated by $n$ quadratic polynomials. The implications of this description for the counting and construction of primary fields are described: an interesting binomial identity underlies one of the construction algorithms. The product on the ring of LWPs can be described as a commutative star product. The quadratic algebra of lowest weight polynomials has a dual quadratic algebra which is non-commutative. We discuss the possible physical implications of this dual algebra.

KeYwords: AdS-CFT Correspondence, Conformal and W Symmetry, Differential and Algebraic Geometry

ARXIV EPRINT: 1806.01085 


\section{Contents}

1 Introduction 1

2 Primary fields from differential constraints and polynomial rings $\quad 3$

2.1 Review: lowest weight states and primaries from differential equations 3

2.2 LWPs and the quotient ring $\mathcal{R} / \mathcal{I} \quad 5$

2.3 Representation theory of $V_{H} \quad 6$

2.4 Solving the center of mass constraint and a polynomial ring with quadratic relations

$\begin{array}{lll}2.5 & V_{0} \oplus V_{H} \text { decomposition of Laplacian constraints } & 10\end{array}$

3 Counting of lowest weight states in $V^{\otimes n} \quad 10$

4 The ring of lowest weights in $V^{\otimes n} \quad 12$

$\begin{array}{lll}4.1 & \text { Exact sequence of modules } & 12\end{array}$

$\begin{array}{ll}4.2 & \text { Exact sequence of vector spaces over } \mathbb{C} \\ \end{array}$

5 Refined counting formulae $\quad 19$

$\begin{array}{lll}5.1 & \text { Refined counting: general } d & 20\end{array}$

5.2 Refined counting: $d=3$ case 21

5.3 Refined counting: the $d=4$ case 22

6 The Confluent Binomial Transform and construction 23

6.1 From resolution to construction: counting without signs 23

6.2 Implementing the construction using the natural inner product 26

$\begin{array}{lll}6.3 & \text { Commutative star product on lowest weight polynomials } & 28\end{array}$

7 Further construction methods for lowest weight polynomials $\quad 30$

$\begin{array}{lll}7.1 & \text { Intersection of kernels of two differential operators } & 30\end{array}$

$\begin{array}{lll}7.2 & \text { Constraints and projectors on } V_{d H} \otimes V_{d H} & 31\end{array}$

7.3 Standard algebraic geometry methods for $\mathcal{R} / \mathcal{I} \quad 34$

8 Discussion and future directions $\quad \mathbf{3 4}$

8.1 Further developing the analogy to tracelessness: a generalization of Brauer algebras 34

8.2 Quadratic algebras and Koszul algebras $\quad 35$

8.3 Future direction: coherence relations between two products 36

$\begin{array}{lll}\text { A } & \text { The invariant in } V_{H} \otimes V_{H} \otimes V_{H} & 37\end{array}$

A.1 The $\kappa$ polynomial $\quad 38$

B Examples of $\mathcal{R} / \mathcal{I}$ at low $n, d \quad 40$ 
D Refined counting of LWPs in $d=3$ dimensions: tables

\section{Introduction}

The counting and construction of primary fields in free scalar field theories was found to have surprisingly simple and elegant geometrical structures in $[1,2]$. General primary fields in scalar field theory in $d$ dimensions, which are composites of $n$ elementary fields, are in 1-1 correspondence with polynomials in $n d$ variables, $x_{\mu}^{I}$ where $1 \leq \mu \leq d, 1 \leq I \leq n$ which solve a system of linear first and second order partial differential equations, and obey an invariance condition under $S_{n}$, the symmetric group of permutations of $n$ distinct objects. A holomorphic sector of primaries corresponds to the ring of functions on an $S_{n}$ orbifold. In [3] it was observed that the space of all primary fields in a scalar theory corresponds to a quotient ring, and that this ring also arises in the classification of effective actions.

At the core of these developments is a simple problem in the representation theory of the $d$-dimensional conformal algebra $s o(d, 2)$ and its surprisingly rich connections to polynomial rings, modules over these rings, the standard mathematics of algebraic geometry, as well as to non-commutative algebras and their quotients. According to the operatorstate correspondence in conformal field theory, local operators are in 1-1 correspondence with quantum states. Corresponding to an elementary scalar field in $d$ dimensions and its non-vanishing derivatives is an irreducible representation $V$ of $s o(d, 2)$. The problem is to decompose the tensor product $V^{\otimes n}$ into irreducible representations of $s o(d, 2) \times S_{n}$. A convenient realization of the representation $V$ is in terms of polynomials in $x_{\mu}$ while for $V^{\otimes n}$ we have polynomials in $x_{\mu}^{I}$. This problem can be approached in two steps: find all the states in $V^{\otimes n}$ annihilated by the special conformal transformation generators $K_{\mu}$, then decompose these states according to representations of $s o(d) \times S_{n}$. Further projecting to the trivial representation of $S_{n}$ gives the primary fields for free scalar field theory. The states annihilated by $K_{\mu}$ are the states of lowest conformal dimension in irreducible representations of $s o(d, 2)$, which may have non-trivial $s o(d)$ transformation properties. With the polynomial realization of $V^{\otimes n}$ in hand, these states are certain polynomials in $\mathbb{C}\left[x_{\mu}^{I}\right]$, which we call lowest weight polynomials (LWPs). Following $[1,2]$ we review the fact that LWPs in $\mathbb{C}\left[x_{\mu}^{I}\right]$ are solutions of a system of first and second order partial differential equations. We explain the 1-1 correspondence between the polynomials and the elements of a quotient ring defined in [3]. The first order equations take the form of a condition of vanishing centre of mass momentum. They can be solved explicitly, leading to a description as a polynomial ring in $(n-1) d$ variables $X_{\mu}^{A}$, with $1 \leq A \leq(n-1)$ and transforming in the irreducible representation of $S_{n}$ corresponding to the hook-shaped Young diagram $[n-1,1]$ with first row of length $n-1$ and second row of length 1 . We will denote this irrep 
as $V_{H}$ in the following. Our first new result (section 2.4) is to give an explicit description of the quotient ring in dimension $d$ in terms of $(n-1) d$ generators and explicit quadratic relations. The quadratic relations are given in terms of a Clebsch-Gordan decomposition problem for $S_{n}$, which we explicitly solve.

The Hilbert series of the quotient ring, which can be deduced from the character of $V^{\otimes n}$ implies counting formulae for the number of linearly independent LWPs at each degree in $\mathbb{C}\left[X_{\mu}^{A}\right]$. These dimensions are expressed as an alternating sum of positive quantities. A transform, which we dub the confluent binomial transform, is found which gives the dimensions as sums of positive quantities (section 6). This leads directly to a construction algorithm for the lowest weight polynomials, which we refer to as the first construction algorithm. This is our second main result. We compare this with two additional construction algorithms. Construction II works directly in $\mathbb{C}\left[x_{\mu}^{I}\right]$ and imposes first and second order conditions. Construction III exploits the quadratic constraints and looks at an intersection of projectors. It exploits analogies between the construction of LWPs and the construction of traceless tensors of $s o(k)$, and as such it has links to Brauer algebras which arise as commutants of $s o(k)$ in tensor spaces.

The paper is organized as follows. Section 2 starts with a review of [1-3]. We describe the system of first and second order partial differential equations for polynomials in $\mathbb{C}\left[x_{\mu}^{I}\right]$ which define the LWPs. We explain the correspondence with a quotient ring obtained by quotienting out an ideal $\mathcal{I}$ generated by linear and quadratic constraints. We then establish a description of LWPs where we have solved the first order constraints. This leads to a quotient ring of $\mathbb{C}\left[X_{\mu}^{A}\right]$ by quadratic relations. These quadratic relations are given explicitly in terms of Clebsch-Gordan coefficients for $V_{H} \otimes V_{H}$.

In section 3 we use the $s o(d, 2)$ character of $V^{\otimes n}$ to arrive at the Hilbert series of the ring $\mathcal{L}$ of lowest weight primaries. Section 4 explains the exact sequences of modules over the polynomial ring $\mathcal{R}=\mathbb{C}\left[X_{\mu}^{A}\right]$, which give a resolution of $\mathcal{L}$. This exact sequence implies the Hilbert series. It also leads directly to exact sequences of vector spaces over $\mathbb{C}$. Section 5 extracts counting formulae for LWPs, refined according to $s o(d) \times S_{n}$ irreps, which follow from the exact sequences.

With an understanding of refined counting formulae, we expect to deduce algorithms for construction of LWPs. One tricky point is that the counting formulae in section 5 involve alternating sums of dimensions of vector spaces. In section 6 we show that the counting formula for dimensions of LWPs obtained from the Hilbert series is equivalent to a formula as a sum of positive constructible quantities. An important feature is that this constructive formula at fixed degree $k$ is expressed in terms of LWPs at lower degrees. Both the positive formula and the alternating sum formula involve binomial coefficients. If we denote by $V_{Q}$ the vector space of quadratic constraints, the alternating sum formula involves dimensions of exterior powers of $V_{Q}$, while the positive formula involves dimensions of symmetric powers of $V_{Q}$. The key identity responsible for this inversion relating the positive and alternating sum formula (6.9) turns out to be a special value of a confluent hypergeometric function. Since the transform involves binomial coefficients, is not the standard binomial transform of combinatorics, and has a connection to the confluent hypergeometric function, we use the name confluent binomial transform. The reader is welcome to suggest a better 
name, with appropriate mathematical justification, which we will consider for our future work on this subject. We follow up the discussion of the positive counting formula by describing a construction algorithm for LWPs, which is implemented in Mathematica. In section 6.3 we show that the product on LWPs, coming from the ring structure in $\mathcal{R} / \mathcal{I}$, can be expressed as a commutative star product based on a decomposition of the space of all polynomials into LWPs and a transverse space.

In section 7 we give two additional construction methods mentioned earlier in the introduction.

Section 8 discusses a number of future research directions related to this work.

\section{Primary fields from differential constraints and polynomial rings}

A key result motivating our study is the observation [2] that primary fields constructed from $n$ copies of a free scalar $\phi$, along with their derivatives, correspond to polynomials in variables $x_{\mu}^{I}$ subject to a system of linear differential constraints and an $S_{n}$ invariance condition. There are $d$ first order differential constraints coming from the lowest weight condition that $K_{\mu}$ annihilates a primary field, as well as $n$ Laplacian conditions, coming from the equation of motion. We also explain, following the statement from [3], that these primary fields are in 1-1 correspondence with elements of a polynomial ring, of which the holomorphic sector forms a Calabi-Yau ring as highlighted in [2]. The first order constraints take the form of a zero centre of mass momentum condition, when we view these polynomials in $x_{\mu}^{I}$ as states in a multi-particle quantum mechanics. They can be solved explicitly. This leads to a formulation of the problem of finding the LWPs as a problem of solving $n$ second order differential constraints acting on polynomials in $(n-1) d$ variables, $X_{\mu}^{A}$, where $1 \leq \mu \leq d, 1 \leq A \leq(n-1)$. The LWPs, now viewed as polynomials in $\mathbb{C}\left[X_{\mu}^{A}\right]$, are in 1-1 correspondence with the elements of a quotient of $\mathbb{C}\left[X_{\mu}^{A}\right]$ by an ideal generated by quadratic polynomials. The explicit form of these quadratic polynomials is given in terms of Clebsch-Gordan coefficients for the couplings between $V_{H} \otimes V_{H}$ and $V_{0} \oplus V_{H}$, where $V_{0}$ is the trivial representation.

\subsection{Review: lowest weight states and primaries from differential equations}

The scalar field and its derivatives form a vector space $V$, which is an irreducible representation of $s o(d, 2)$. This representation is isomorphic to the space of harmonic polynomials in $x_{\mu}$. The connection between the standard action of the conformal group on the fields and the action of differential operators on the polynomials is explained in [2].

The generators of $s o(d, 2)$ form the algebra

$$
\begin{aligned}
{\left[K_{\mu}, P_{\nu}\right] } & =2 M_{\mu \nu}-2 D \delta_{\mu \nu} \\
{\left[D, P_{\mu}\right] } & =P_{\mu} \\
{\left[D, K_{\mu}\right] } & =-K_{\mu} \\
{\left[M_{\mu \nu}, K_{\alpha}\right] } & =\delta_{\nu \alpha} K_{\mu}-\delta_{\mu \alpha} K_{\nu} \\
{\left[M_{\mu \nu}, P_{\alpha}\right] } & =\delta_{\nu \alpha} P_{\mu}-\delta_{\mu \alpha} P_{\nu}
\end{aligned}
$$


The algebra $s o(d, 2)$ is realised on these polynomials as [4]

$$
\begin{aligned}
K_{\mu} & =\frac{\partial}{\partial x_{\mu}} \\
P_{\mu} & =\left(x^{2} \partial_{\mu}-2 x_{\mu} x \cdot \partial-(d-2) x_{\mu}\right) \\
D & =\left(x \cdot \partial+\frac{(d-2)}{2}\right) \\
M_{\mu \nu} & =x_{\mu} \partial_{\nu}-x_{\nu} \partial_{\mu}
\end{aligned}
$$

Thinking of $x_{\mu}$ as the co-ordinates of a particle, this is a single particle representation. The tensor product $V^{\otimes n}$ can be realized on a many-particle space of functions $\Psi\left(x_{\mu}^{I}\right)$, where $1 \leq I \leq n$ labels the particle number. We now have generators

$$
\begin{aligned}
K_{\mu}^{I} & =\frac{\partial}{\partial x_{\mu}^{I}} \\
P_{\mu}^{I} & =\left(\sum_{\rho=1}^{d} x_{\rho}^{I} x_{\rho}^{I} \frac{\partial}{\partial x_{\mu}^{I}}-2 x_{\mu}^{I} \sum_{\rho=1}^{d} x_{\rho}^{I} \frac{\partial}{\partial x_{\rho}^{I}}-(d-2) x_{\mu}^{I}\right)
\end{aligned}
$$

The generators of the diagonal $s o(d, 2)$ acting as a sum of the generators on each tensor factor of $V^{\otimes n}$ are

$$
\begin{aligned}
K_{\mu} & =\sum_{I} K_{\mu}^{I} \\
P_{\mu} & =\sum_{I} P_{\mu}^{I}
\end{aligned}
$$

Let $\mathcal{H}$ be the space of harmonic polynomials in $x_{\mu}$. Polynomials in $x_{\mu}^{I}$ which are harmonic in each of the $x_{\mu}^{I}$, i.e. which are annihilated by the $n$ operators

$$
\sum_{\mu=1}^{d} \frac{\partial^{2}}{\partial x_{\mu}^{I} \partial x_{\mu}^{I}}
$$

span the space $\mathcal{H}^{\otimes n}$.

A lowest weight polynomial (LWP) denoted $L\left(x_{\mu}^{I}\right)$ satisfies the equations

$$
\begin{array}{r}
\sum_{I=1}^{n} \frac{\partial L}{\partial x_{\mu}^{I}}=0 \text { for } 1 \leq \mu \leq d \\
\sum_{\mu=1}^{d} \frac{\partial^{2} L}{\partial x_{\mu}^{I} \partial x_{\mu}^{I}}=0 \text { for } 1 \leq I \leq n
\end{array}
$$

The $S_{n}$ invariant lowest weight polynomials correspond to primary fields. We will refer to the first constraint appearing above as the center of mass constraint, for obvious reasons.

In this paper we will focus our attention on LWPs. The projection to $S_{n}$ invariants is a standard exercise, illustrated in concrete examples in [2]. 


\subsection{LWPs and the quotient $\operatorname{ring} \mathcal{R} / \mathcal{I}$}

The polynomial ring $\mathbb{C}\left[x_{\mu}^{I}\right]$ is denoted as $\mathcal{R}$. Consider the ideal $\mathcal{I}$ generated by the $n$ elements $\sum_{\mu=1}^{d} x_{\mu}^{I} x_{\mu}^{I}$ along with the $d$ elements $\sum_{I} x_{\mu}^{I}$. This is denoted by

$$
\mathcal{I}=\left\langle\sum_{\mu=1}^{d} x_{\mu}^{I} x_{\mu}^{I}, \sum_{I} x_{\mu}^{I}\right\rangle
$$

and consists of elements in $\mathcal{R}$ of the form

$$
\sum_{a=1}^{n+d} h_{a} g_{a}
$$

where the $g_{a}$ refer to all the generators in (2.8) and $h_{a}$ are arbitrary elements of the ring $\mathcal{R}$. Following the statement in [3], we will explain that the quotient $\operatorname{ring} \mathcal{R} / \mathcal{I}$ is isomorphic as a vector space over $\mathbb{C}$ to the primaries. $\mathcal{R}, \mathcal{I}$ are vector spaces over $\mathbb{C}$ and the quotient ring $\mathcal{R} / \mathcal{I}$ is also a quotient vector space. Each element is an equivalence class of vectors, related to each other by addition of elements in $\mathcal{I}$. For each lowest weight polynomial satisfying (2.7) there is one such equivalence class. It is useful to explain this correspondence.

Consider the map $\phi: \mathcal{R} \rightarrow \mathcal{H}^{\otimes n}$ defined by

$$
\phi: x_{\mu_{1}}^{I_{1}} x_{\mu_{2}}^{I_{2}} \cdots x_{\mu_{k}}^{I_{k}} \rightarrow P_{\mu_{1}}^{I_{1}} P_{\mu_{2}}^{I_{2}} \cdots P_{\mu_{k}}^{I_{k}}(1)
$$

$\sum_{\mu} x_{\mu}^{I} x_{\mu}^{I}$ are in the kernel of this map since, as is easily checked using the explicit form of $P_{\mu}^{I}$ in $(2.3)$

$$
\sum_{\mu=1}^{d} P_{\mu}^{I} P_{\mu}^{I}(1)=0
$$

The representation $\mathcal{H}^{\otimes n}$ is, by construction, a reducible lowest weight representation of $s o(d, 2) .1$ is a lowest weight state for $\mathrm{SO}(d, 2)^{\times n}$ annihilated by $K_{\mu}^{I}$ for all $I \in\{1,2, \cdots, n\}$. The irrep $\mathcal{H}_{n+k, j_{1}, j_{2}}$ contains lowest weight states under the diagonal $\mathrm{SO}(d, 2)$ (annihilated by $K_{\mu}=\sum_{I} K_{\mu}^{I}$ ) of dimension $\Delta=n\left(\frac{d-2}{2}\right)+k$ and transforming in the rank $k$ traceless symmetric tensor irrep of $s o(d)$. There will be a multiplicity for each lowest weight state. This is expressed by introducing a vector space of multiplicities $\mathcal{M}_{k, j_{1}, j_{2}}$. Thus, we can write

$$
\mathcal{H}^{\otimes n}=\bigoplus_{k, j_{1}, j_{2}} \mathcal{H}_{n+k, j_{1}, j_{2}} \otimes \mathcal{M}_{k, j_{1}, j_{2}}
$$

For classification of the irreps of $s o(d, 2)$ and their character formulae, see [5] and refs therein. A lowest weight state with $\Delta=n+k$ generates a tower of states at higher $\Delta$ through the action of $P_{\mu}=\sum_{I} P_{\mu}^{I}$. These descendants themselves form a subspace that can be characterized as follows

$$
\text { Descendants }=\operatorname{Span}\left(\mathcal{P}\left(\left\{P_{\mu}^{I}\right\}\right) P_{\mu}(1)\right)
$$

where $\mathcal{P}\left(\left\{P_{\mu}^{I}\right\}\right)$ is any polynomial in the $P_{\mu}^{I}$. These correspond, under the map $\phi$ to the ideal generated by $x_{\mu}=\sum_{I} x_{\mu}^{I}$. The quotient space $\mathcal{H}^{\otimes n} /$ Descendants is equivalent, as a 
vector space, to the space of lowest weight states

$$
\mathcal{L}=\bigoplus_{k, j_{1}, j_{2}} \mathcal{M}_{k, j_{1}, j_{2}}
$$

Now consider the homomorphism $\phi$ as a map from $\mathcal{R}$ to $\mathcal{H}^{\otimes n} /$ Descendants. The kernel of this map is the ideal in $\mathcal{R}$ given by the ideal $\mathcal{I}$ in (2.8). This shows that

$$
\mathcal{L}=\mathcal{H}^{\otimes n} / \text { Descendants }=\mathcal{R} / \mathcal{I}
$$

Equality here means isomorphism, as graded vector spaces over $\mathbb{C}$.

\subsection{Representation theory of $V_{H}$}

The $I$ index of $x_{\mu}^{I}$, ranging over $1 \leq I \leq n$, transforms in the natural representation, $V_{\text {nat }}$ of $S_{n}$. This representation has an orthogonal decomposition into irreducible representations

$$
V_{\text {nat }}=V_{0} \oplus V_{H}
$$

$V_{0}$ is the one-dimensional representation. $V_{H}$ has dimension $(n-1)$ and corresponds to the Young diagram $[n-1,1]$ with row lengths $n-1,1$. The tensor product $V_{H} \otimes V_{H}$ can be decomposed into irreducible representations as

$$
V_{H} \otimes V_{H}=V_{0} \oplus V_{H} \oplus V_{[n-2,2]} \oplus V_{[n-2,1,1]}
$$

The explicit Clebsch-Gordan coefficients for $V_{0}$ and $V_{H}$ will turn out to be useful in obtaining a new description of the ring defined earlier in section 2.2, where the linear constraints have been solved.

Let us write

$$
V_{\text {nat }}=\operatorname{Span}\left\{e_{1}, e_{2}, \cdots, e_{n}\right\}
$$

and introduce the inner product

$$
\left\langle e_{I}, e_{J}\right\rangle=\delta_{I J}
$$

The $S_{n}$ action on $V_{\text {nat }}$ is

$$
D^{\text {nat }}(\sigma) e_{I}=e_{\sigma^{-1}(I)}
$$

and obeys the homomorphism property

$$
D^{\text {nat }}\left(\sigma_{1}\right) D^{\text {nat }}\left(\sigma_{2}\right)=D^{\text {nat }}\left(\sigma_{1} \sigma_{2}\right)
$$

The inner product (2.19) is invariant under the $S_{n}$ action. The linear combination

$$
e_{0}=\frac{1}{\sqrt{n}} \sum_{I=1}^{n} e_{I}
$$

is invariant, normalized to 1 and spans $V_{0}$. We can choose a convenient orthonormal basis for $V_{H}$ as

$$
e_{A}=\frac{1}{\sqrt{A(A+1)}}\left(e_{1}+e_{2}+\cdots+e_{A}-A e_{A+1}\right)
$$


for $A \in\{1,2, \cdots, n-1\}$. Introducing the notation $S_{A I}$ for these coefficients we have

$$
e_{A}=\sum_{I=1}^{n} S_{A I} e_{I}
$$

for $A \in\{1,2, \cdots, n-1\}$, and

$$
S_{A I}=\frac{1}{\sqrt{A(A+1)}}\left(-A \delta_{I, A+1}+\sum_{J=1}^{A} \delta_{J, I}\right)
$$

It is also useful to introduce extend $A$ to $A \in\{0,1, \cdots, n-1\}$, so that

$$
\begin{aligned}
e_{A=0} & =\frac{1}{\sqrt{n}}\left(e_{1}+e_{2}+\cdots+e_{n}\right) \\
S_{0 I} & =\frac{1}{\sqrt{n}} \quad \text { for } \quad 1 \leq I \leq n
\end{aligned}
$$

We have the orthonormality relations

$$
\sum_{I=1}^{n} S_{A I} S_{B I}=\delta_{A B}
$$

This expresses the orthonormality of states $e_{A}, A \in\{1, \cdots, n-1\}$ within $V_{H}$, and within $V_{0}$ for $A, B=0$, as well as the orthogonality of all the states in $V_{H}$ with the invariant state in $V_{0}$. We also have

$$
\sum_{A=0}^{n-1} S_{A I} S_{A J}=\delta_{I J}
$$

Given these orthogonality relations, the inverse transformation expressing $e_{I}$ in terms of the $e_{A}$ are

$$
e_{I}=\sum_{A=0}^{n-1} S_{A I} e_{A}
$$

The following sum will play a crucial role in our subsequent treatment of the ring defined in section 2.2

$$
\kappa_{A B C}=\sum_{I=1}^{n} S_{C I} S_{A I} S_{B I}
$$

Let $D_{C C^{\prime}}^{H}(\sigma)$ denote the matrix representing the permutation $\sigma$ in the hook representation $H$. Note that $\kappa_{A B C}$ has the following $S_{n}$ invariance property.

$$
\begin{aligned}
\kappa_{A B C} & \left.\left.\left.=\sum_{I=1}^{n}\langle H, C| \text { nat, } I\right\rangle\langle H, B| \text { nat, } I\right\rangle\langle H, A| \text { nat, } I\right\rangle \\
& \left.\left.\left.=\sum_{I=1}^{n}\langle H, C| \text { nat, } \sigma(I)\right\rangle\langle H, B| \text { nat, } \sigma(I)\right\rangle\langle H, A| \text { nat, } \sigma(I)\right\rangle \\
& =\sum_{I=1}^{n} \sum_{A^{\prime}, B^{\prime}, C^{\prime}=1}^{n-1} D_{C C^{\prime}}^{H}(\sigma) D_{B B^{\prime}}^{H}(\sigma) D_{A A^{\prime}}^{H}(\sigma) S_{C^{\prime} I} S_{A^{\prime} I} S_{B^{\prime} I} \\
& =\sum_{A^{\prime}, B^{\prime}, C^{\prime}=1}^{n-1} D_{C C^{\prime}}^{H}(\sigma) D_{B B^{\prime}}^{H}(\sigma) D_{A A^{\prime}}^{H}(\sigma) \kappa_{A^{\prime} B^{\prime} C^{\prime}}
\end{aligned}
$$


This shows that $\kappa_{A B C}$ is a state in $V_{H} \otimes V_{H} \otimes V_{H}$ which is invariant under the simultaneous linear transformation of the three states by $S_{n}$. We know there is, up to normalization, precisely one such state, since $V_{H}$ appears once in the Clebsch-Gordan decomposition of $V_{H} \otimes V_{H}$. Equivalently $V_{0}$ appears once in $V_{H} \otimes V_{H} \otimes V_{H}$. We conclude that $\kappa_{A B C}$ is this Clebsch-Gordan coefficient.

In appendix A we calculate this invariant explicitly to get

$$
\begin{aligned}
\kappa_{A B C}= & -A B C \delta_{A, B, C}+B C \delta_{B, C} \Theta(B<A)+A B \delta_{A, B} \Theta(A<C)+A C \delta_{A, C} \Theta(A<B) \\
& -C \Theta(C<A) \Theta(C<B)-B \Theta(B<A) \Theta(B<C)-A \Theta(A<C) \Theta A<B) \\
& +\operatorname{Min}(A, B, C)
\end{aligned}
$$

$\Theta(B<A)$ is defined to be 1 if $B<A$ and 0 otherwise. We also find that

$$
\begin{aligned}
\kappa\left(z_{A}\right) & \equiv \sum_{A, B, C=1}^{n-1} \kappa_{A B C} z_{A} z_{B} z_{C} \\
& =\sum_{A} A\left(1-A^{2}\right) z_{A}^{3}+\sum_{A<B} 3 A(1+A) z_{A}^{2} z_{B}
\end{aligned}
$$

and

$$
\begin{aligned}
\kappa_{A}(z) & =\sum_{B, C} \kappa_{A B C} z_{B} z_{C} \\
& =A\left(1-A^{2}\right) z_{A}^{2}+\sum_{B: B<A} B(1+B) z_{B}^{2}+\sum_{B: A<B} 2 A(1+A) z_{B} z_{A}
\end{aligned}
$$

\subsection{Solving the center of mass constraint and a polynomial ring with quadratic relations}

In solving the constraints that determine the LWPs, a fruitful approach is to solve the center of mass constraint (COM) and only then consider the remaining constraints in (2.7). This approach exploits the $S_{n}$ structure of the problem. We will use the elements of $S_{n}$ representation theory from section 2.3 .

As noted earlier, the $I$ index transforms in the natural representation of $S_{n}$ which has a decomposition into irreducibles as

$$
V_{\text {nat }}=V_{0} \oplus V_{H}
$$

We will use the coefficients $S_{A I}$ for this decomposition introduced in section 2.3 to define

$$
X_{\mu}^{A}=\sum_{I=1}^{n} S_{A I} x_{\mu}^{I}
$$

$X_{\mu}^{0}$ is invariant under $S_{n}$. The $X_{\mu}^{A}$ for $1 \leq A \leq n-1$ form an orthonormal basis of states in $V_{H}$.

The COM condition is satisfied by setting

$$
X_{\mu}^{0}=0
$$


The inverse transformation, following (2.29), is

$$
x_{\mu}^{I}=\sum_{A=0}^{n-1} S_{A I} X_{\mu}^{A}
$$

The quadratic conditions can be expressed as

$$
\sum_{A, B=0}^{n-1} \sum_{\mu=1}^{d} S_{A I} S_{B I} X_{\mu}^{A} X_{\mu}^{B}=0
$$

The linear COM conditions (2.37) imply the quadratic conditions become

$$
\sum_{A, B=1}^{n-1} \sum_{\mu=1}^{d} S_{A I} S_{B I} X_{\mu}^{A} X_{\mu}^{B}=0
$$

It is useful to express this in the $V_{0} \oplus V_{H}$ basis. Towards this end, multiply by $S_{C I}$ and sum over $I$ to find

$$
Q_{C} \equiv \sum_{I=1}^{n} \sum_{A, B=1}^{n-1} \sum_{\mu=1}^{d} S_{C I} S_{A I} S_{B I} X_{\mu}^{A} X_{\mu}^{B}=0
$$

For $C=0$, we get

$$
Q_{0}=\sum_{A} \sum_{\mu=1}^{d} X_{\mu}^{A} X_{\mu}^{A}=0
$$

For $C>0$ we have

$$
\sum_{\mu=1}^{d} \sum_{A, B=1}^{n-1} \kappa_{C A B} X_{\mu}^{A} X_{\mu}^{B}=0
$$

Note that, while the $A, B$ indices range over $\{1, \cdots, n-1\}$, the $C$ index ranges over $\{0,1, \cdots, n-1\}$. Given the explicit formulae stated in section 2.3 and derived in appendix A, the quadratic constraints can be expressed as

For $1 \leq A \leq(n-1)$ :

$$
\begin{aligned}
& A\left(1-A^{2}\right) \sum_{\mu=1}^{d} X_{\mu}^{A} X_{\mu}^{A}+\sum_{B: B>A} \sum_{\mu=1}^{d} 2 A(1+A) X_{\mu}^{A} X_{\mu}^{B}+\sum_{B: B<A} \sum_{\mu=1}^{d} B(1+B) X_{\mu}^{B} X_{\mu}^{B}=0 \\
& \text { and } \\
& \sum_{A=1}^{n-1} \sum_{\mu=1}^{d} X_{\mu}^{A} X_{\mu}^{A}=0
\end{aligned}
$$

The upshot is that we have a description of the construction of the primaries as the construction of polynomials in the hook variables $X_{\mu}^{A}$ with $1 \leq A \leq n-1,1 \leq \mu \leq d$, subject to the quadratic constraints (2.44). In appendix B we study the variety defined by these quadratic constraints for some low values of $n, d$, and compute the associated Hilbert series using Sage. It is in complete agreement with our counting of LWPs. 


\section{5 $V_{0} \oplus V_{H}$ decomposition of Laplacian constraints}

The LWPs solve the $n$ Laplacian conditions

$$
\sum_{\mu} \frac{\partial^{2} F}{\partial x_{\mu}^{I} \partial x_{\mu}^{I}}=0
$$

These $n$ conditions transform in the natural representation $V_{\text {nat }}$ of $S_{n}$. We can again move to the $V_{0} \oplus V_{H}$ basis as follows

$$
\square_{C}=\sum_{I=1}^{n} \sum_{\mu=1}^{d} S_{C I} \frac{\partial^{2}}{\partial x_{\mu}^{I} \partial x_{\mu}^{I}}
$$

Now expand the $x$-derivatives in terms of $X$-derivatives, and use the fact that we are acting on translation invariant functions to drop derivatives with respect to $X_{\mu}^{0}$. We have

$$
\sum_{I=1}^{n} \sum_{\mu=1}^{d} S_{C I} \frac{\partial^{2}}{\partial x_{\mu}^{I} \partial x_{\mu}^{I}}=\sum_{I} \sum_{A, B=1}^{n-1} \sum_{\mu=1}^{d} S_{C I} S_{A I} S_{B I} \frac{\partial^{2}}{\partial X_{\mu}^{A} \partial X_{\mu}^{B}}
$$

Notice that the quantity

$$
\kappa_{A B C}=\sum_{I=1}^{n} S_{C I} S_{A I} S_{B I}
$$

introduced in the previous section, has appeared above.

There are $n-1$ linear combinations of the Laplacians which transform as $V_{H}$, given by

$$
\square_{C}=\sum_{A, B=1}^{n-1} \sum_{\mu=1}^{d} \kappa_{A B C} \frac{\partial^{2}}{\partial X_{\mu}^{A} \partial X_{\mu}^{B}}
$$

Together with the $S_{n}$ invariant Laplacian

$$
\square_{0}=\sum_{\nu=1}^{d} \sum_{A=1}^{n-1} \frac{\partial^{2}}{\partial x_{\nu}^{A} \partial x_{\nu}^{A}}
$$

we have $n$ differential operators acting on the functions of $X_{\mu}^{A}$.

In summary, we have now arrived at a description of LWPs as polynomial functions in $X_{\mu}^{A}$ i.e. functions on

$$
\left(\mathbb{R}^{d}\right)^{(n-1)}
$$

subject to the $n$ Laplacian conditions in (2.49) and (2.50). The LWPs are dual to primary operators in the free CFT.

\section{Counting of lowest weight states in $V^{\otimes n}$}

$V$ is the representation of $s o(d, 2)$ collecting all the states which correspond, by the operator-state correspondence, to a single scalar field and its derivatives. Above we have established that the lowest weight states in $V^{\otimes n}$ form a polynomial ring. We will develop 
this description further in this section by counting these lowest weight states. Specifically, we give a formula for the generating function of the number of lowest weight states in $V^{\otimes n}$, at weight $\Delta=n\left(\frac{d-2}{2}\right)+k$. The $S_{n}$ invariant states among these lowest weight states are the primaries.

In $d$ dimensions, the character of the free scalar field $s o(d, 2)$ irrep is

$$
\chi_{V}(s)=t r s^{\Delta}=s^{(d-2) / 2} \frac{\left(1-s^{2}\right)}{(1-s)^{d}}
$$

The character for $V^{\otimes n}$, a reducible representation, is then

$$
\chi_{V \otimes n}(s)=\left(\chi_{V}(s)\right)^{n}=s^{n(d-2) / 2} \frac{\left(1-s^{2}\right)^{n}}{(1-s)^{n d}}
$$

The trace of $s^{\Delta}$ over states obtained by acting with momenta on a lowest weight state (annihilated by $K_{\mu}$ ) with $\Delta=n(d-2) / 2+k$ is

$$
\frac{s^{n(d-2) / 2+k}}{(1-s)^{d}}
$$

Let the multiplicities of these lowest weight states in $V^{\otimes n}$ be $\mathcal{N}_{k}$. To determine the multiplicities $\mathcal{N}_{k}$ we expand $\chi_{V \otimes n}(s)$ in terms of the traces in (3.3) as follows

$$
\chi_{V^{\otimes n}}(s)=s^{n(d-2) / 2} \frac{\left(1-s^{2}\right)^{n}}{(1-s)^{n d}}=\sum_{k=0}^{\infty} \mathcal{N}_{k} \frac{s^{n(d-2) / 2+k}}{(1-s)^{d}}
$$

Hence the generating function for the multiplicities of lowest weight states is

$$
\begin{aligned}
\sum_{k=0}^{\infty} \mathcal{N}_{k} s^{k} & =\frac{\left(1-s^{2}\right)^{n}}{(1-s)^{d(n-1)}} \\
& =\frac{1-n s^{2}+\frac{n(n-1)}{2} s^{4}+\cdots}{(1-s)^{d(n-1)}} \\
& =\frac{1}{(1-s)^{d(n-1)}} \sum_{k=0}^{n}(-1)^{k}\left(\begin{array}{l}
n \\
k
\end{array}\right) s^{2 k}
\end{aligned}
$$

In this result we can already recognize elements of our discussion from section 2 appearing. Indeed, the denominator of the Hilbert series given above shows that there are $d(n-1)$ generators in the ring. These are the $X_{\mu}^{A}$. The numerator implies that at quadratic order, we have $n$ relations. These are the constraints (2.42) and (2.43). Note that $\left(\begin{array}{l}n \\ k\end{array}\right)$ is the dimension of the $k$-fold anti-symmetric product of $V_{Q}$, the $n$ dimensional space spanned by the quadratic constraints $Q_{A}$. An important point for the discussion in the next section is that

$$
\frac{1}{(1-s)^{d(n-1)}}\left(\begin{array}{l}
n \\
k
\end{array}\right) s^{2 k}
$$

is the trace of $s^{\Delta}$ over $\mathcal{R} \otimes \Lambda^{k}\left(V_{Q}\right)$. Finally, it is worth noting that the counting function in the first line of (3.5) is palindromic. 


\section{The ring of lowest weights in $V^{\otimes n}$}

In the previous section we have obtained the counting function for the lowest weights in $V^{\otimes n}$. These lowest weights form a polynomial ring. The counting function for the ring is a rational function. The ring is a quotient of the polynomial ring, by an ideal. The ideal is generated by $n$ quadratic expressions.

The structure of the counting function can be explained using the theory of Hilbert series, in terms of the relations between the generators of the ideal, relations between these relations and so on. This notion of generators, relations and relations between relations is made precise in the theory of Hilbert series in terms of exact sequences of modules over the polynomial ring. References we found useful include [6-8].

In this section we will describe the relevant exact sequence and show that it matches the counting function derived from $s o(d, 2)$ representation theory. We then explain how the exact sequence of modules of the polynomial ring $\mathcal{R}$ leads to exact sequences of vector spaces over the base field $\mathbb{C}$. These exact sequences are used to derive a refined counting formula for $s o(d) \times S_{n}$ irreps among the lowest weights. The $s o(d)$ scalar lowest weights are of interest in effective field theory [3].

\subsection{Exact sequence of modules}

We will consider the following exact sequence of modules over $\mathcal{R}=\mathbb{C}\left[X_{\mu}^{A}\right]$

$$
0 \stackrel{f_{0}}{\longrightarrow} \mathcal{R} \otimes \Lambda^{n}\left(V_{Q}\right) \stackrel{f_{n}}{\longrightarrow} \cdots \rightarrow \mathcal{R} \otimes \Lambda^{2}\left(V_{Q}\right) \stackrel{f_{2}}{\longrightarrow} \mathcal{R} \otimes V_{Q} \stackrel{f_{1}}{\longrightarrow} \mathcal{R} \stackrel{f_{\mathcal{R}}}{\longrightarrow} \mathcal{L} \stackrel{f_{\mathcal{L}}}{\longrightarrow} 0
$$

The tensor products are defined over the base field $\mathbb{C}$. Elements of $\mathcal{R} \otimes V_{Q}$ are given by

$$
\sum_{A=0}^{n-1} h_{A} \otimes Q_{A}
$$

where $h_{A} \in \mathcal{R}$. The map $f_{1}$ acts as

$$
f_{1}: \sum_{A=0}^{n-1} h_{A} \otimes Q_{A} \rightarrow \sum_{A=0}^{n-1} h_{A} Q_{A}
$$

Its image is the ideal generated by $Q_{A}$. This ideal $\mathcal{I}(d, n)$, consists of elements in the ring $\mathcal{R}=\mathbb{C}\left[X_{\mu}^{A}\right]$ of the form

$$
\sum_{A=0}^{n-1} h_{A} Q_{A}
$$

where $h_{A}$ are general elements in $\mathcal{R}(d, n)$.

$\mathcal{R} \otimes V_{Q}$ is a module for $\mathcal{R}$. An element $h \in \mathcal{R}$ acts as

$$
\sum_{A=0}^{n-1} h_{A} \otimes Q_{A} \rightarrow \sum_{A=0}^{n-1} h h_{A} \otimes Q_{A}
$$

Since $\mathcal{L}$ is the quotient $\mathcal{R} / \mathcal{I}$ and the map $f_{\mathcal{L}}$ takes all the elements of $\mathcal{L}$ to 0 , the image of $f_{\mathcal{R}}$ is the kernel of $f_{\mathcal{L}}$. Thus the sequence is exact at $\mathcal{R}$ and $\mathcal{L}$. 
The elements of $\mathcal{R} \otimes \Lambda^{I}\left(V_{Q}\right)$ are

$$
\epsilon^{A_{1}, \cdots, A_{I}, A_{I+1}, \cdots, A_{n}} h_{A_{1} A_{2} \cdots A_{I}} \otimes Q_{A_{1}} \otimes Q_{A_{2}} \cdots \otimes Q_{A_{I}}
$$

(the repeated indices $A_{I+1}, \cdots, A_{n}$ are summed). Under the map $f_{I}$, they go to

$$
\epsilon^{A_{1}, \cdots, A_{I}, A_{I+1}, \cdots, A_{n}} h_{A_{1} A_{2} \cdots A_{I}} Q_{A_{1}} \otimes Q_{A_{2}} \cdots \otimes Q_{A_{I}}
$$

Under the composite map $f_{I} \circ f_{I-1}$, we have

$$
\begin{gathered}
f_{I} \circ f_{I-1}: \epsilon^{A_{1}, \cdots, A_{I}, A_{I+1}, \cdots, A_{n}} h_{A_{1} A_{2} \cdots A_{I}} \otimes Q_{A_{1}} \otimes Q_{A_{2}} \cdots \otimes Q_{A_{I}} \rightarrow \\
\quad \rightarrow \epsilon^{A_{1}, \cdots, A_{I}, A_{I+1}, \cdots, A_{n}} h_{A_{1} A_{2} \cdots A_{I}} Q_{A_{1}} Q_{A_{2}} \otimes Q_{A_{3}} \cdots \otimes Q_{A_{I}}=0
\end{gathered}
$$

This shows that

$$
\operatorname{Im}\left(f_{I}\right) \subseteq \operatorname{Ker}\left(f_{I-1}\right)
$$

Thus we have established that the image of $f_{I}$ is in the kernel of $f_{I-1}$. For exactness we need to show that the image of $f_{I}$ is equal to the kernel of $f_{I-1}$. This will follow, by additionally proving that $\operatorname{Ker}\left(f_{I-1}\right) \subseteq \operatorname{Im}\left(f_{I}\right)$.

To proceed further, motivated by the analysis in Chapter 4 of [9] (where exactness is proved for a sequence involving $\operatorname{Sym}^{k}(V) \otimes \Lambda^{k}(V)$, in the context of proving that $\operatorname{Sym}(V)$ and $\Lambda(V)$ are Koszul algebras), we introduce two operators. The first operator, $d$, is a re-expression of the maps $f_{I}$ introduced above. We define

$$
d=\sum_{A=0}^{n-1} Q_{A} \otimes \iota_{A}
$$

where $Q_{A}$ acts by multiplying any polynomial $f$ in $\mathcal{R}$ by $\sum_{\mu=1}^{d} \kappa_{A B C} X_{\mu}^{B} X_{\mu}^{C}$ and $\iota_{A}$ is interior multiplication with $Q_{A}$

$$
\begin{aligned}
\iota_{A}\left(Q_{B_{1}} \wedge \cdots \wedge Q_{B_{i}}\right) & =\sum_{k=1}^{i}(-1)^{k-i} Q_{B_{1}} \wedge \cdots \wedge \hat{Q}_{B_{k}} \wedge \cdots \wedge Q_{B_{i}} \text { if } A=B_{k} \\
& =0 \quad A \notin\left\{B_{1}, B_{2}, \cdots, B_{i}\right\}
\end{aligned}
$$

It is simple to demonstrate, using this definition, that $d^{2}=0$, which is equivalent to the discussion above that lead to the conclusion (4.9). To motivate the second operator we need, we employ a decomposition of polynomials that will be derived in section 6.2: the space of degree $d$ polynomials can be decomposed as

$$
p^{(d)}=p_{h}^{(d)}+Q_{A} p_{h, A}^{(d-2)}+Q_{A} Q_{B} p_{h, A B}^{(d-4)}+\cdots
$$

with the coefficients $p_{h}^{(d)}, p_{h, A}^{(d-2)}, p_{h, A B}^{(d-4)}$ all annihilated by $(C=0,1, \ldots, n-1)$.

$$
\square_{C}=\sum_{A, B=1}^{n-1} \sum_{\mu=1}^{d} \kappa_{C A B} \frac{\partial^{2}}{\partial X_{\mu}^{A} \partial X_{\mu}^{B}}
$$


The repeated index $A$ in the second term is summed, as are the $A, B$ in the third term, etc. The decomposition (4.12) is unique in the sense that the coefficients $p_{h}^{(d)}, p_{h, A}^{(d-2)}, p_{h, A B}^{(d-4)}, \ldots$, in the expansion are unique. The second operator we use is

$$
\alpha=\sum_{A=0}^{n-1} d_{A} \otimes \psi_{A}
$$

where

$$
\psi_{A}(g)=g \wedge Q_{A}
$$

and the action of $d_{A}$ is defined using the expansion (4.12): $d_{A}$ simply removes a $Q_{A}$ from each term and it annihilates $p_{h}^{(d)}$ :

$$
d_{A}\left(p_{h}^{(d)}+Q_{B} p_{h, B}^{(d-2)}+Q_{B} Q_{C} p_{h, B C}^{(d-4)}+\cdots\right)=p_{h, A}^{(d-2)}+2 Q_{B} p_{h, A B}^{(d-4)}+\cdots
$$

Again, from these definitions it is easy to see that $\alpha^{2}=0$ : applying $d_{A}$ twice produces a symmetric two index tensor $p_{h, A B C \cdots D}^{q} Q_{C} \cdots Q_{D}$ which vanishes when summed against $g \wedge Q_{A} \wedge Q_{B}$, which is antisymmetric in $A, B$.

We will now argue that $\alpha \circ d+d \circ \alpha$ is not degenerate, that is, it has an inverse. In fact, we will show that acting on a monomial of degree $t$ in the $Q \mathrm{~s}$, it is proportional to the identity

$$
\alpha \circ d+d \circ \alpha=1+t
$$

In this case, acting on any element $k_{h, A B \cdots E}^{(q)} Q_{A} Q_{B} \cdots Q_{E} \otimes Q_{A_{1}} \wedge \cdots \wedge Q_{A_{i}}$ in the kernel of $d$, we have

$$
\begin{gathered}
(\alpha \circ d+d \circ \alpha) k_{h, A B \cdots E}^{(q)} Q_{A} Q_{B} \cdots Q_{E} \otimes Q_{A_{1}} \wedge \cdots \wedge Q_{A_{i}} \\
\quad=d \circ \alpha\left(k_{h, A B \cdots E}^{(q)} Q_{A} Q_{B} \cdots Q_{E} \otimes Q_{A_{1}} \wedge \cdots \wedge Q_{A_{i}}\right) \\
=d\left(\alpha\left(k_{h, A B \cdots E}^{(q)} Q_{A} Q_{B} \cdots Q_{E} \otimes Q_{A_{1}} \wedge \cdots \wedge Q_{A_{i}}\right)\right) \\
=(1+t) k_{h, A B \cdots E}^{(q)} Q_{A} Q_{B} \cdots Q_{E} \otimes Q_{A_{1}} \wedge \cdots \wedge Q_{A_{i}}
\end{gathered}
$$

so that

$$
\operatorname{Ker}\left(f_{I-1}\right) \subseteq \operatorname{Im}\left(f_{I}\right)
$$

and hence, together with (4.9) we have exactness

$$
\operatorname{Ker}\left(f_{I-1}\right)=\operatorname{Im}\left(f_{I}\right)
$$

Let us now complete the argument by showing that $\alpha \circ d+d \circ \alpha$ is indeed not degenerate. For $d \circ \alpha$ we find (assume that $p_{h, A B \cdots E}^{(q)}$ has $t$ indices $A B \cdots E$ )

$$
\begin{aligned}
& d \circ \alpha\left(p_{h, A B \cdots E}^{(q)} Q_{A} Q_{B} \cdots Q_{E} \otimes Q_{A_{1}} \wedge \cdots \wedge Q_{A_{i}}\right) \\
& =d\left(\sum_{S \notin\left\{A_{1}, \ldots, A_{i}\right\}} t p_{h, S B \cdots E}^{(q)} Q_{B} \cdots Q_{E} \otimes Q_{A_{1}} \wedge \cdots \wedge Q_{A_{i}} \wedge Q_{S}\right)
\end{aligned}
$$




$$
\begin{aligned}
= & \sum_{S \notin\left\{A_{1}, \ldots, A_{i}\right\}} t Q_{S} p_{h, S B \cdots E}^{(q)} Q_{B} \cdots Q_{E} \otimes Q_{A_{1}} \wedge \cdots \wedge Q_{A_{i}} \\
& +\sum_{S \notin\left\{A_{1}, \ldots, A_{i}\right\}} \sum_{k=1}^{i}(-1)^{i-k+1} t Q_{A_{k}} p_{h, S B \cdots E}^{(q)} Q_{B} \cdots Q_{E} \otimes Q_{A_{1}} \wedge \cdots \hat{Q}_{A_{k}} \cdots \wedge Q_{A_{i}} \wedge Q_{S}
\end{aligned}
$$

For $\alpha \circ d$ we have

$$
\begin{aligned}
\alpha \circ d\left(p_{h, A B \cdots E}^{(q)} Q_{A} Q_{B} \cdots Q_{E} \otimes Q_{A_{1}} \wedge \cdots \wedge Q_{A_{i}}\right) \\
=\alpha\left(\sum_{k=1}^{i}(-1)^{i-k} p_{h, A B \cdots E}^{(q)} Q_{A} Q_{B} \cdots Q_{E} Q_{A_{k}} \otimes Q_{A_{1}} \wedge \cdots \hat{Q}_{A_{k}} \cdots \wedge Q_{A_{i}}\right) \\
=\sum_{k=1}^{i} \sum_{S \notin\left\{A_{1}, \ldots, \hat{A}_{k}, \ldots, A_{i}\right\}}(-1)^{i-k} d_{S}\left(p_{h, A B \cdots E}^{(q)} Q_{A} Q_{B} \cdots Q_{E} Q_{A_{k}}\right) \otimes Q_{A_{1}} \wedge \cdots \hat{Q}_{A_{k}} \cdots \wedge Q_{A_{i}} \wedge Q_{S} \\
=\sum_{k=1}^{i} \sum_{S \notin\left\{A_{1}, \ldots, \hat{A}_{k}, \ldots, A_{i}\right\}}(-1)^{i-k} t p_{h, S B \cdots E}^{(q)} Q_{B} \cdots Q_{E} Q_{A_{k}} \otimes Q_{A_{1}} \wedge \cdots \hat{Q}_{A_{k}} \cdots \wedge Q_{A_{i}} \wedge Q_{S} \\
\quad+p_{h, A B \cdots E}^{(q)} Q_{A} Q_{B} \cdots Q_{E} \otimes Q_{A_{1}} \wedge \cdots \wedge Q_{A_{i}}
\end{aligned}
$$

The second term in the answer (4.21) cancels against the first term in (4.22) to leave

$$
\sum_{k=1}^{i} t p_{h, A_{k} B \cdots E}^{(q)} Q_{B} \cdots Q_{E} Q_{A_{k}} \otimes Q_{A_{1}} \wedge \cdots \wedge Q_{A_{i}}
$$

Thus, we find

$$
\begin{aligned}
(d \circ \alpha+\alpha \circ d)\left(p_{h, A B \cdots E}^{(q)} Q_{A} Q_{B} \cdots Q_{E} \otimes Q_{A_{1}} \wedge \cdots \wedge Q_{A_{i}}\right) \\
=\sum_{S \notin\left\{A_{1}, \ldots, A_{i}\right\}} t p_{h, S B \cdots E}^{(q)} Q_{S} Q_{B} \cdots Q_{E} \otimes Q_{A_{1}} \wedge \cdots \wedge Q_{A_{i}} \\
\quad+\sum_{k=1}^{i} t p_{h, A_{k} B \cdots E}^{(q)} Q_{B} \cdots Q_{E} Q_{A_{k}} \otimes Q_{A_{1}} \wedge \cdots \wedge Q_{A_{i}} \\
\quad+p_{h, A B \cdots E}^{(q)} Q_{A} Q_{B} \cdots Q_{E} \otimes Q_{A_{1}} \wedge \cdots \wedge Q_{A_{i}} \\
=(1+t) p_{h, A B \cdots E}^{(q)} Q_{A} Q_{B} \cdots Q_{E} \otimes Q_{A_{1}} \wedge \cdots \wedge Q_{A_{i}}
\end{aligned}
$$

which is the result we wanted.

Finally, note that the above long exact sequence can be thought of as consisting of several short exact sequences. One is the standard short exact sequence for quotients

$$
0 \rightarrow \mathcal{I} \rightarrow \mathcal{R} \rightarrow \mathcal{R} / \mathcal{I} \rightarrow 0
$$

The next is

$$
\operatorname{Syz}(\mathcal{I}) \rightarrow \mathcal{R} \otimes V_{Q} \rightarrow \mathcal{I}
$$

Here a basis for $V_{Q}$ gives the generators of $\mathcal{I}$. $\operatorname{Syz}(\mathcal{I})$ is the syzygy module for $\mathcal{I}$. It is generated by $\Lambda^{2}\left(V_{Q}\right)$ so that its generators and relations are expressed in a sequence

$$
\operatorname{Syz}(\operatorname{Syz}(\mathcal{I})) \rightarrow \mathcal{R} \otimes \Lambda^{2}\left(V_{Q}\right) \rightarrow \operatorname{Syz}(\mathcal{I})
$$




\subsection{Exact sequence of vector spaces over $\mathbb{C}$}

We can consider the vector space formed by polynomials of a fixed degree. Since the modules of the last section are defined over the ring, a single exact sequence of modules implies, upon specializing to fixed degree, an exact sequence for each of these vector spaces. The polynomials at fixed $n$ and fixed degree $k$ are polynomials dual to primaries constructed using $n$ fields and $k$ derivatives. There is therefore an interesting CFT motivation to consider the exact sequences between the vector spaces formed by polynomials of a fixed degree, which is the goal of this section. The $X_{\mu}^{A}$ transform as $V_{d} \otimes V_{H}$ of $s o(d) \times S_{n}$, where $V_{d}$ is the $d$-dimensional vector of $s o(d)$ and $V_{H}$ is the $(n-1)$ dimensional hook representation of $S_{n}$. For convenience, we define $V_{d H}=V_{d} \otimes V_{H}$. Polynomials of degree $k$ in $X_{\mu}^{A}$ form a vector space over $\mathbb{C}$ isomorphic to the space of rank $k$ symmetric tensors, denoted $\operatorname{Sym}^{k}\left(V_{d H}\right)$.

At $k=2$ we have

$$
0 \rightarrow V_{Q} \rightarrow \operatorname{Sym}^{2}\left(V_{d H}\right) \rightarrow \mathcal{L}(2, d, n) \rightarrow 0
$$

The space of LWPs at $k=2$, denoted $\mathcal{L}(2, d, n)$, is obtained by setting the $Q_{A}$ 's to zero. The space $\operatorname{Sym}^{2}\left(V_{d H}\right)$ is the space of degree two polynomials in the hook variables $X_{\mu}^{A}$. The $Q_{A}$ 's form a subspace of $\operatorname{Sym}^{2}\left(V_{d H}\right)$, so we have a map from $V_{Q}$ to $\operatorname{Sym}^{2}\left(V_{d H}\right)$. The definition of $\mathcal{L}(2, d, n)$ as the quotient space ensures that the image of the first map is exactly the kernel of the second map, so that the sequence above is exact. Denoting the dimension of $\operatorname{Sym}^{k}\left(V_{d H}\right)$ by $S(k, d, n)$, we know that

$$
S(k, d, n)=\frac{((n-1) d+k-1) !}{k !((n-1) d-1) !} \equiv S(k, D=d(n-1))
$$

The second equality emphasizes the fact that this depends only on $D=d(n-1)$. The exact sequence (4.28) implies the following formula for the dimension of the space of LWPs

$$
L(2, d, n)=S(2, d, n)-\operatorname{Dim}\left(V_{Q}\right)
$$

It is simple to check this independently by comparing to the coefficient of $s^{2}$ in the expansion of $(3.5)$.

Next, consider degree $k=4$. The relevant exact sequence is

$$
0 \rightarrow \Lambda^{2}\left(V_{Q}\right) \rightarrow \operatorname{Sym}^{2}\left(V_{d H}\right) \otimes V_{Q} \rightarrow \operatorname{Sym}^{4}\left(V_{d H}\right) \rightarrow \mathcal{L}(4, d, n) \rightarrow 0
$$

as we explain below. Start by introducing the map $f$ defined by

$$
f: \operatorname{Sym}^{2}\left(V_{d H}\right) \otimes V_{Q} \rightarrow \operatorname{Sym}^{4}\left(V_{d H}\right)
$$

Concretely, we have

$$
f: Q_{A} \otimes X_{\mu_{1}}^{a_{1}} X_{\mu_{2}}^{a_{2}} \rightarrow Q_{A} X_{\mu_{1}}^{a_{1}} X_{\mu_{2}}^{a_{2}}=\sum_{\mu=1}^{d} \kappa_{A B C} X_{\mu}^{B} X_{\mu}^{C} X_{\mu_{1}}^{a_{1}} X_{\mu_{2}}^{a_{2}}
$$

Thus, under this map we find

$$
f: Q_{A} \otimes Q_{B}-Q_{B} \otimes Q_{A} \rightarrow 0
$$


Thus, the kernel of the map is $\Lambda^{2}\left(V_{Q}\right)$. When we have a 4 -term exact sequence as above the far right vector space is the cokernel, i.e.

$$
\mathcal{L}(4, d, n)=\operatorname{Sym}^{4}\left(V_{d H}\right) / \operatorname{Im}(f)
$$

This is indeed the definition of $\mathcal{L}(4, d, n)$ : it is the quotient space of the degree 4 polynomials in $X_{\mu}^{A}$ obtained by setting to zero anything of the form $Q X X$. The exact sequence (4.31) implies the following relation

$$
L(4, d, n)=S(4, d, n)-S(2, d, n) \operatorname{Dim}\left(V_{Q}\right)+\operatorname{Dim} \Lambda^{2}\left(V_{Q}\right)
$$

which agrees with the coefficient of $s^{4}$ in the expansion of (3.5).

These exact sequences generalize to any $k$. We have

$$
\begin{aligned}
0 & \rightarrow \operatorname{Sym}^{k-2 L}\left(V_{d H}\right) \otimes \Lambda^{L}\left(V_{Q}\right) \rightarrow \cdots \rightarrow \operatorname{Sym}^{k-2 I}\left(V_{d H}\right) \otimes \Lambda^{I}\left(V_{Q}\right) \rightarrow \cdots \\
& \rightarrow \operatorname{Sym}^{k-2}\left(V_{d H}\right) \otimes V_{Q} \rightarrow \operatorname{Sym}^{k}\left(V_{d H}\right) \rightarrow \mathcal{L}(k, d, n) \rightarrow 0
\end{aligned}
$$

where $L=\min \left(\left\lfloor\frac{k}{2}\right\rfloor, n\right)$. If $k$ is even and $k / 2 \leq n$, then the second term in the sequence is $\Lambda^{k / 2}\left(V_{Q}\right)$. If $k / 2 \geq n$, it is $\operatorname{Sym}^{k-2 n}\left(V_{d H}\right) \otimes \Lambda^{n}\left(V_{Q}\right)$. If $k$ is odd and $(k-1) / 2 \leq n$, then the first non-trivial term is $V_{d H} \otimes \Lambda^{\frac{k-1}{2}}\left(V_{Q}\right)$. If $n \leq(k-1) / 2$, then it is $\operatorname{Sym}^{k-2 n}\left(V_{d H}\right) \otimes \Lambda^{n}\left(V_{Q}\right)$.

One basic building block that the above sequences are constructed from is the following

$$
\begin{aligned}
\cdots \rightarrow \operatorname{Sym}^{k-2 I}\left(V_{d H}\right) \otimes \Lambda^{I}\left(V_{Q}\right) & \rightarrow \operatorname{Sym}^{k+2-2 I}\left(V_{d H}\right) \otimes \Lambda^{I-1}\left(V_{Q}\right) \\
& \rightarrow \operatorname{Sym}^{k+4-2 I}\left(V_{d H}\right) \otimes \Lambda^{I-2}\left(V_{Q}\right) \cdots
\end{aligned}
$$

A simple generalization of the discussion above gives the maps required for this basic building block. First note that the space $\Lambda^{I-1}\left(V_{Q}\right)$ is spanned by

$$
\epsilon^{A_{1} \cdots A_{I-1} A_{I} \cdots A_{L}} Q_{A_{1}} \otimes Q_{A_{2}} \otimes \cdots \otimes Q_{A_{I-1}}
$$

with $L=\min \left(\left\lfloor\frac{k}{2}\right\rfloor, n\right)$ as above. Define the map $f$ which maps

$$
f: \operatorname{Sym}^{k+2-2 I}\left(V_{d H}\right) \otimes \Lambda^{I-1}\left(V_{Q}\right) \rightarrow \operatorname{Sym}^{k+4-2 I}\left(V_{d H}\right) \otimes \Lambda^{I-2}\left(V_{Q}\right)
$$

as follows

$$
\begin{aligned}
& f\left(X_{\mu_{1}}^{a_{1}} \cdots X_{\mu_{k+2-2 I}}^{a_{k+2-2 I}} \otimes \epsilon^{A_{1} \cdots A_{I-1} A_{I} \cdots A_{L}} Q_{A_{1}} \otimes Q_{A_{2}} \otimes \cdots \otimes Q_{A_{I-1}}\right) \\
& \quad=\sum_{\mu=1}^{d} X_{\mu_{1}}^{a_{1}} \cdots X_{\mu_{k+2-2 I}}^{a_{k+2-2 I}} \kappa_{A_{I-1} B C} X_{\mu}^{B} X_{\mu}^{C} \otimes \epsilon^{A_{1} \cdots A_{I-1} A_{I} \cdots A_{L}} Q_{A_{1}} \otimes Q_{A_{2}} \otimes \cdots \otimes Q_{A_{I-2}}
\end{aligned}
$$

It is clear that the image of the map

$$
g: \operatorname{Sym}^{k-2 I}\left(V_{d H}\right) \otimes \Lambda^{I}\left(V_{Q}\right) \rightarrow \operatorname{Sym}^{k+2-2 I}\left(V_{d H}\right) \otimes \Lambda^{I-1}\left(V_{Q}\right)
$$

is in the kernel of $f$. Indeed, the image of $g$ is spanned by

$$
\sum_{\mu=1}^{d} X_{\mu_{1}}^{a_{1}} \cdots X_{\mu_{k-2 I}}^{a_{k-2 I}} \kappa_{A_{I} B C} X_{\mu}^{B} X_{\mu}^{C} \otimes \epsilon^{A_{1} \cdots A_{I-1} A_{I} \cdots A_{L}} Q_{A_{1}} \otimes Q_{A_{2}} \otimes \cdots \otimes Q_{A_{I-1}}
$$


Under $f$ this maps to zero

$$
\begin{aligned}
& f\left(\sum_{\mu=1}^{d} X_{\mu_{1}}^{a_{1}} \cdots X_{\mu_{k-2 I}}^{a_{k-2 I}} \kappa_{A_{I} B C} X_{\mu}^{B} X_{\mu}^{C} \otimes \epsilon^{A_{1} \cdots A_{I-1} A_{I} \cdots A_{L}} Q_{A_{1}} \otimes Q_{A_{2}} \otimes \cdots \otimes Q_{A_{I-1}}\right) \\
& \quad=\sum_{\mu, \nu=1}^{d} X_{\mu_{1}}^{a_{1}} \cdots X_{\mu_{k-2 I}}^{a_{k-2 I}} \kappa_{A_{I} B C} X_{\mu}^{B} X_{\mu}^{C} \kappa_{A_{I-1} F G} X_{\nu}^{F} X_{\nu}^{G} \otimes \\
& \epsilon^{A_{1} \cdots A_{I-1} A_{I} \cdots A_{L}} Q_{A_{1}} \otimes Q_{A_{2}} \otimes \cdots \otimes Q_{A_{I-2}}=0
\end{aligned}
$$

where the last equality follows because

$$
\sum_{\mu, \nu=1}^{d} \kappa_{A_{I} B C} X_{\mu}^{B} X_{\mu}^{C} \kappa_{A_{I-1} F G} X_{\nu}^{F} X_{\nu}^{G}
$$

is symmetric under swapping $A_{I}$ and $A_{I-1}$, and it is contracted with $\epsilon^{A_{1} \cdots A_{L}}$. To complete the discussion, consider

$$
\cdots \rightarrow \operatorname{Sym}^{k-2}\left(V_{d H}\right) \otimes V_{Q} \rightarrow \operatorname{Sym}^{k}\left(V_{d H}\right) \rightarrow \mathcal{L}(k, d, n) \rightarrow 0
$$

In terms of the map $h$ which maps

$$
h: \operatorname{Sym}^{k-2}\left(V_{d H}\right) \otimes V_{Q} \rightarrow \operatorname{Sym}^{k}\left(V_{d H}\right)
$$

we have $\mathcal{L}(k, d, n)=\operatorname{Sym}^{k}\left(V_{d H}\right) / \operatorname{Im}(h)$, which is true since $\mathcal{L}(k, d, n)$ is the quotient space of the degree $k$ polynomials in the $X_{\mu}^{A}$ obtained by setting anything of the form $Q X \cdots X$ to zero.

The argument above shows that the image is in the kernel. To establish exactness, we need to show that the kernel is equal to the image. This can be done exactly as we did it in section 4.1. We again introduce $d$ (again motivated by the mappings we just discussed) and $\alpha$, defined precisely as we did above. The generalization of the argument is then obvious and we will not repeat it here.

The exact sequences we have presented in this section imply that

$$
L(k, d, n)=\sum_{I=0}^{\min \left(\left\lfloor\frac{k}{2}\right\rfloor, n\right)}(-1)^{I} \operatorname{Dim}\left(\operatorname{Sym}^{k-2 I}\left(V_{d H}\right)\right) \operatorname{Dim}\left(\Lambda^{I}\left(V_{Q}\right)\right)
$$

This formula will be used in the next section to refine the counting of LWPs, by keeping track of the $s o(d) \times S_{n}$ irreps of the LWPs. To understand why this refined counting is possible, note that the maps involved in the exact sequences given in this section, all commute with $s o(d) \times S_{n}$. The maps involved in the vector space exact sequences involve replacing $Q_{A}$ by its explicit form $\kappa_{A B C} X_{\mu}^{B} X_{\mu}^{C}$. Since the spacetime indices are fully contracted, the maps replaces an $s o(d)$ scalar with an $s o(d)$ scalar. Similarly, since $\kappa_{A B C}$ is an invariant tensor, the map is from the hook to the hook irrep. Since this refinement holds for all of the vector space exact sequences, it should hold for the module exact sequences too. This is indeed clear from the expressions $d=\sum_{A} Q_{A} \otimes \iota_{A}$ used in section 4.1. 


\section{Refined counting formulae}

We have managed to count the number of LWPs of fixed degree, or equivalently, lowest weight states in $V^{\otimes n}$. There are good reasons to refine this counting using the $s o(d) \times S_{n}$ symmetry present in the problem. Primaries in the free field theory are $S_{n}$ invariants. They are labeled by their dimension and $s o(d)$ representation property. In addition, $s o(d)$ scalars are relevant for identifying possible terms in the Lagrangian of effective field theory [3]. This refined counting will ultimately lead to a construction algorithm for the LWPs. In this section we will carry out this refined counting, using the formula (4.47) which follows from the exact sequences developed in the last section.

A useful starting point for the refined counting is (4.47) which we re-write slightly here, by substituting $V_{Q} \rightarrow V_{\text {nat }}$

$$
L(k, d, n)=\sum_{I=0}^{\min \left(\left\lfloor\frac{k}{2}\right\rfloor, n\right)}(-1)^{I} \operatorname{Dim}\left(\operatorname{Sym}^{k-2 I}\left(V_{d H}\right)\right) \operatorname{Dim}\left(\Lambda^{I}\left(V_{\text {nat }}^{\left(S_{n}\right)}\right)\right)
$$

We can start by writing

$$
\operatorname{Sym}^{k-2 I}\left(V_{d H}\right)=\bigoplus_{\Lambda_{1}, \Lambda_{3,1} \vdash n} V_{\Lambda_{1}}^{(s o(d))} \otimes V_{\Lambda_{3,1}}^{\left(S_{n}\right)} \otimes V_{\Lambda_{1}, \Lambda_{3,1}}
$$

The r.h.s. is the decomposition of $\operatorname{Sym}^{k-2 I}\left(V_{d H}\right)$ in terms of irreducible representations of $s o(d) \times S_{n}$, labeled by $\left(\Lambda_{1}, \Lambda_{3,1}\right)$. $\Lambda_{3,1}$ is a partition of $n$. A basis in terms of irreps will include a multiplicity label for the pair $\left(\Lambda_{1}, \Lambda_{3,1}\right)$, this multiplicity space is denoted by $V_{\Lambda_{1}, \Lambda_{3,1}}$.

We will denote the dimensions of these multiplicity spaces by $\operatorname{Mult}\left(\left(\operatorname{Sym}^{k-2 I}\left(V_{d H}\right) ; \Lambda_{1}^{s o(d)} \otimes \Lambda_{3,1}^{\left(S_{n}\right)}\right)\right.$. We can also decompose the antisymmetric (wedge) product of $n$ copies of the natural representation $\Lambda^{I}\left(V_{\text {nat }}^{\left(S_{n}\right)}\right)$ into irreps of $S_{n}$. The number of times a given $S_{n}$ irrep $\Lambda_{3,2}$ appears will be denoted by $\operatorname{Mult}\left(\Lambda^{I}\left(V_{\text {nat }}^{\left(S_{n}\right)}\right) ; \Lambda_{3,2}^{\left(S_{n}\right)}\right)$. We can now write the $s o(d) \times S_{n}$ refined version of (5.1) as

$$
\begin{gathered}
L\left(\Lambda_{1}^{s o(d)}, \Lambda_{3}^{\left(S_{n}\right)} ; k, d, n\right) \\
=\sum_{\Lambda_{3,1}, \Lambda_{3,2} \vdash n} \sum_{\substack{I=0 \\
\times \operatorname{mun}\left(\left\lfloor\frac{k}{2}\right\rfloor, n\right)}}(-1)^{I} \operatorname{Mult}\left(\Lambda^{I}\left(V_{\text {nat }}^{\left(S_{n}\right)}\right) ; \Lambda_{3,2}^{\left(S_{n}\right)}\right) C\left(\Lambda_{3,1}, \Lambda_{3,2} ; \Lambda_{3}\right)
\end{gathered}
$$

$C\left(\Lambda_{3,1}, \Lambda_{3,2} ; \Lambda_{3}\right)$ is the Kronecker multiplicity for $\Lambda_{3,1} \otimes \Lambda_{3,2} \rightarrow \Lambda_{3}$. The 1.h.s. is the multiplicity of irreps $\Lambda_{1}, \Lambda_{3}$ in the space of lowest weight states of dimension $L(k, d, n)$. Consequently, we have

$$
L(k, d, n)=\sum_{\Lambda_{1}, \Lambda_{3}} \operatorname{Dim}_{s o(d)}\left(\Lambda_{1}\right) \operatorname{Dim}_{S_{n}}\left(\Lambda_{3}\right) L\left(\Lambda_{1}^{s o(d)}, \Lambda_{3}^{\left(S_{n}\right)} ; k, d, n\right)
$$

It is important to note that the alternating sum formula (5.1) for $L(k, d, n)$ does not, by itself, imply the refined formula (5.3). However, the exact sequences underlying (5.1), 
alongside the fact discussed in section 4 that the maps in this exact sequence are $s o(d) \times S_{n}$ invariant, do imply that the sequences can be restricted to specific irreps and hence imply the refined counting formulae.

We will now make (5.3) more explicit to produce some general $s o(d) \times S_{n}$ refined counting formulae for the space of lowest weight states. To determine how many times irrep $\Lambda_{3,2}$ appears in $\Lambda^{I}\left(V_{\text {nat }}\right)$, we take the trace of the projector to $\Lambda_{3,2}$ from $\Lambda^{I}\left(V_{\text {nat }}\right)$. The result is

$$
\begin{aligned}
\operatorname{Mult} & \left(\Lambda^{I}\left(V_{\mathrm{nat}}^{\left(S_{n}\right)}\right) ; \Lambda_{3,2}^{\left(S_{n}\right)}\right) \\
= & \sum_{p \vdash n} \sum_{q \vdash I}(-1)^{q_{2}+q_{4}+\cdots} \frac{\chi_{\Lambda_{3,2}}^{p}}{\operatorname{Sym}(p) \operatorname{Sym}(q)} \prod_{i=1}^{I}\left(\sum_{d \mid i} d p_{d}\right)^{q_{i}}
\end{aligned}
$$

where $\chi_{\Lambda_{3,2}}$ is the $S_{n}$ character of the permutation with cycle structure $p$ in irrep $\Lambda_{3,2}$ and

$$
\operatorname{Sym}(p)=\prod_{i} i^{p_{i}} p_{i} !
$$

with $p_{i}$ denoting the number of parts in partition $p$ that are equal to $i$. This is obtained by setting $\Lambda_{2}=\left[1^{k}\right]$ in (5.14) of appendix C, and using the fact that characters in the anti-symmetric are given by $(-1)^{q_{2}+q_{4}+\cdots}$. We now need to consider refining $\operatorname{Sym}^{k}\left(V_{d H}\right)$. For the $s o(d)$ part, we can use $s o(d)$ characters. For $d=3$, we have $s o(3)$, so that we only need well known $s u(2)$ results. For $d=4$, we will use $s o(4)=s u(2) \times s u(2)$, so again we need only $s u(2)$ results.

\subsection{Refined counting: general $d$}

We can make the formula (5.3) more explicit for general $d$. The formulae we write here will be not be as computationally efficient as for $d=3,4$ but may still be useful in further studies of $s o(d, 2)$ representations and free field primaries in higher dimensions. We focus on the $d$-dependent quantity

$$
\operatorname{Mult}\left(\operatorname{Sym}^{k}\left(V_{d H}\right) ; \Lambda_{1}^{s o(d)} \otimes \Lambda_{3}^{\left(S_{n}\right)}\right)
$$

in (5.3). $\operatorname{Sym}^{k}\left(V_{d H}\right)$ is the $S_{k}$ invariant part of $\left(V_{d} \otimes V_{H}\right)^{\otimes k}=V_{d}^{\otimes k} \otimes V_{H}^{\otimes k}$. We have the decompositions

$$
V_{d}^{\otimes k}=\bigoplus_{\Lambda_{1}, \Lambda_{2}} V_{\Lambda_{1}}^{s o(d)} \otimes V_{\Lambda_{2}}^{\left(S_{k}\right)} \otimes V_{\Lambda_{1}, \Lambda_{2}}
$$

and

$$
V_{H}^{\otimes k}=\bigoplus_{\Lambda_{3}, \Lambda_{4}} V_{\Lambda_{3}}^{\left(S_{n}\right)} \otimes V_{\Lambda_{4}}^{\left(S_{k}\right)} \otimes V_{\Lambda_{3}, \Lambda_{4}}
$$

To count $S_{k}$ invariants in $V_{d H}^{\otimes k}$, we use the above while setting $\Lambda_{4}=\Lambda_{2}$. This leads to

$\operatorname{Mult}\left(\operatorname{Sym}^{k}\left(V_{d H}, \Lambda_{1}^{s o(d)} \otimes \Lambda_{3}^{\left(S_{n}\right)}\right)\right)=\sum_{\Lambda_{2} \vdash k} \operatorname{Mult}\left(V_{d}^{\otimes k}, \Lambda_{1}^{s o(d)} \otimes \Lambda_{2}^{\left(S_{k}\right)}\right) \operatorname{Mult}\left(V_{H}^{\otimes k}, \Lambda_{3}^{\left(S_{n}\right)} \otimes \Lambda_{2}^{\left(S_{k}\right)}\right)$ 
The $\operatorname{Mult}\left(V_{d}^{\otimes k}, \Lambda_{1}^{s o(d)} \otimes \Lambda_{2}^{\left(S_{k}\right)}\right)$ can be calculated using characters of $s o(d)$ and $S_{k}$.

$$
\begin{aligned}
\operatorname{Mult} & \left(V_{d}^{\otimes k}, V_{\Lambda_{1}}^{s o(d)} \otimes \Lambda_{2}^{\left(S_{k}\right)}\right) \\
& =\frac{1}{k !} \sum_{\sigma \in S_{k}} \int d U \chi_{\Lambda_{1}}(U) \chi_{\Lambda_{2}}(\sigma) \operatorname{tr}_{V_{d}^{\otimes k}}\left(U^{\otimes k} \sigma\right) \\
& =\sum_{p \vdash k} \int d U \chi_{\Lambda_{1}}(U) \frac{\chi_{\Lambda_{2}}^{p}}{\operatorname{Symp}} \prod_{i}\left(t r U^{i}\right)^{p_{i}}
\end{aligned}
$$

For the cases of $d=3,4$ we will give expressions below in terms of generating functions, which are more explicit than the group integrals above.

\subsection{Refined counting: $d=3$ case}

We need the multiplicities of $V_{\Lambda_{1}^{s o(3)}} \otimes V_{\Lambda_{2}^{\left(s_{k}\right)}}$ in $V_{3}^{\otimes k}$. This problem has been considered in [10]. Our coordinates $X_{\mu}^{A}$ are in the 3 of $s o(3)$, which is the spin 1 of $\mathrm{SU}(2)$. For $d=3$, $\Lambda_{1}$ is parameterised by one integer $l$ for the spin. Our multiplicities are given by formula 6.2 of [10], with $m=2$ for spin 1 . The result is

$$
\begin{aligned}
& \operatorname{Mult}\left(V_{3}^{\otimes k},[l] \otimes \Lambda_{2}\right) \\
& \quad=\text { Coefficient }\left(q^{0},(1-q) q^{\sum_{i} c_{i}\left(c_{i}-1\right) / 2+l / 2-k} \prod_{(i, j) \in \Lambda_{2}} \frac{\left(1-q^{3-i+j}\right)}{\left(1-q^{h(i, j)}\right)}\right)
\end{aligned}
$$

where the notation is an instruction to pick up the coefficient of $q^{0}$ in the expansion of the second argument above. $c_{i}$ is the length of the $i$ 'th column of $\Lambda_{2} . k$ is the number of boxes in $\Lambda_{2}$. $(i, j)$ label the row and column of the boxes in $\Lambda_{2}$ and $h(i, j)$ is the hook length of the box. The multiplicity $\left.\operatorname{Mult}\left(\operatorname{Sym}^{k-2 I}\left(V_{d H}\right) ;\left(\Lambda_{1}^{s o(d)}=[l]\right) \otimes \Lambda_{3,1}^{\left(S_{n}\right)}\right)\right)$ which appears in the present $s o(3)$ instance of (5.1) can be made more explicit. We use $V_{d H}=V_{d}^{s o(d)} \otimes V_{H}^{S_{n}}$ so that the $k$ fold tensor power is

$$
\left(V_{d H}\right)^{\otimes k-2 I}=\left(V_{d}^{\otimes k-2 I} \otimes V_{H}^{\otimes k-2 I}\right)
$$

We can decompose the $s o(d)$ and $S_{n}$ parts separately into irreps of $s o(d) \times S_{k}$ and $S_{n} \times S_{k}$ respectively. Identifying the $S_{k}$ irreps and summing projects to the invariant of $S_{k}$. The outcome is

$$
\begin{aligned}
& \left.\operatorname{Mult}\left(\operatorname{Sym}^{k-2 I}\left(V_{d H}\right) ;\left(\Lambda_{1}^{s o(d)}=[l]\right) \otimes \Lambda_{3,1}^{\left(S_{n}\right)}\right)\right) \\
& \quad=\sum_{\Lambda_{2} \vdash k-2 I} \operatorname{Mult}\left(V_{3}^{\otimes k-2 I},[l] \otimes \Lambda_{2}\right) \operatorname{Mult}\left(V_{H}^{\otimes k-2 I}, V_{\Lambda_{3,1}}^{\left(S_{n}\right)} \otimes V_{\Lambda_{2}}^{\left(S_{k-2 I}\right)}\right)
\end{aligned}
$$

where

$$
\operatorname{Mult}\left(V_{H}^{\otimes k-2 I}, V_{\Lambda_{3,1}}^{\left(S_{n}\right)} \otimes V_{\Lambda_{2}}^{\left(S_{k-2 I}\right)}\right)=\sum_{p \vdash n} \sum_{q \vdash k} \frac{\chi_{\Lambda_{1}}^{p} \chi_{\Lambda_{2}}^{q}}{\operatorname{Sym}(p) \operatorname{Sym}(q)} \prod_{i=1}^{k}\left(-1+\sum_{d \mid i} d p_{d}\right)^{q_{i}}
$$

We have again used appendix C. Explicit counting results obtained by implementing the formulas of this section in Sage are given in appendix D. 


\subsection{Refined counting: the $d=4$ case}

The fundamental of $\mathrm{SO}(4)$ is the $V_{1 / 2} \otimes \bar{V}_{1 / 2}$ of $S U_{L}(2) \times S U_{R}(2)$, where $V_{1 / 2}$ is the twodimensional spin half irrep of $\mathrm{SU}(2) . X_{\mu}^{A}$ transforming in $V_{4} \otimes V_{H}$ can be written as $X_{\alpha, \dot{\alpha}}^{A}$ to reflect the description as $V_{1 / 2} \otimes V_{1 / 2} \otimes V_{H}$. It is useful to think of $V_{1 / 2}$ as a two-dimensional rep of $\mathrm{U}(2)$, which we call $U_{L}(2)$ and likewise $\bar{V}_{1 / 2}$ as an irrep of $U_{R}(2)$.

The decomposition relevant for our discussion is

$$
V_{1 / 2}^{\otimes k}=\bigoplus_{\Lambda_{2,1} \in Y_{2}^{(k)}} V_{\Lambda_{2,1}}^{u_{L}(2)} \otimes V_{\Lambda_{2,1}}^{\left(S_{k}\right)}
$$

where $Y_{2}^{(k)}$ is the set of Young diagrams with $k$ boxes and at most two rows. This decomposition is an example of Schur-Weyl duality (see for example [11]). The $u(2)$ irrep associated with a Young diagram having row lengths $\left(r_{1}, r_{2}\right)$ has $s u(2) \operatorname{spin}\left(r_{1}-r_{2}\right) / 2$ and dimension $r_{1}-r_{2}+1$. Similarly we have

$$
\bar{V}_{1 / 2}^{\otimes k}=\bigoplus_{\Lambda_{2,2} \in Y_{2}^{(k)}} V_{\Lambda_{2,2}}^{u_{R}(2)} \otimes V_{\Lambda_{2,2}}^{\left(S_{k}\right)}
$$

For the $k^{\prime}$ th power of the hook we have

$$
V_{H}^{\otimes k}=\bigoplus_{\substack{\Lambda_{3} \in Y^{(n)} \\ \Lambda_{2,3} \in Y^{(k)}}} V_{\Lambda_{3}}^{\left(S_{n}\right)} \otimes V_{\Lambda_{2,3}}^{\left(S_{k}\right)} \otimes V_{\Lambda_{3}, \Lambda_{2,3}}
$$

The dimension of $V_{\Lambda_{3}, \Lambda_{2,3}}$ is the multiplicity of the irrep $V_{\Lambda_{3}}^{\left(S_{n}\right)} \otimes V_{\Lambda_{2,3}}^{\left(S_{k}\right)}$ in the tensor product. The dimension is given by equation (5.14). The final result is

$$
\operatorname{Sym}^{k}\left(V_{4 H}\right)=\bigoplus_{\substack{\Lambda_{3} \in Y^{(n)} \\ \Lambda_{2,1} \in Y_{2}^{(k)} \\ \\ \Lambda_{2,2} \in Y_{2}^{(k)} \\ \\ \Lambda_{2,3} \in Y_{2}^{(k)}}} V_{\Lambda_{2,1}}^{u_{L}(2)} \otimes V_{\Lambda_{2,2}}^{u_{R}(2)} \otimes V_{\Lambda_{3}}^{\left(S_{n}\right)} \otimes V_{\Lambda_{3}, \Lambda_{2,3}} \otimes V_{\Lambda_{2,1}, \Lambda_{2,2}, \Lambda_{2,3}}^{S_{k} \text { invts }}
$$

where $V_{\Lambda_{2,1}, \Lambda_{2,2}, \Lambda_{2,3}}^{S_{k} \text { invts }}$ is the space of $S_{k}$ invariants in the Kronecker product $\Lambda_{2,1} \otimes \Lambda_{2,2} \otimes \Lambda_{2,3}$ of $S_{k}$ irreps. The multiplicity of irrep $\Lambda_{3}^{\left(S_{n}\right)},\left(l_{1}, l_{2}\right)_{s o(4)}$ appearing in $\operatorname{Sym}^{k}\left(V_{4 H}\right)$ is thus

$$
\begin{aligned}
& \sum_{\Lambda_{2,1} \in Y_{2}^{(k)}} \sum_{\Lambda_{2,2} \in Y_{2}^{(k)}} \sum_{\Lambda_{2,3} \in Y^{(k)}} \delta\left(r_{1}\left(\Lambda_{2,1}\right)-r_{2}\left(\Lambda_{2,1}\right), l_{1}\right) \delta\left(r_{1}\left(\Lambda_{2,2}\right)-r_{2}\left(\Lambda_{2,2}\right), l_{2}\right) \\
& \quad \times \operatorname{Mult}\left(V_{\Lambda_{2,3}}^{\left(S_{k}\right)} \otimes V_{\Lambda_{3}}^{\left(S_{n}\right)}, V_{H}^{\otimes k}\right) C\left(\Lambda_{2,1}, \Lambda_{2,2}, \Lambda_{2,3}\right)
\end{aligned}
$$

$C$ is the Kronecker coefficient for $S_{k}$. We can plug this into (5.3) in order to get the refined counting for $d=4$. Counting results obtained from these formulas using Sage are given in appendix E. 


\section{The Confluent Binomial Transform and construction}

The exact sequences we derived in section 4 have led to a dimension formula for $\mathcal{L}(k, d, n)$ (or for $\mathcal{I}(k, d, n)$ ) as an alternating sum involving exterior powers of $V_{Q}$. In this section we will show that there is a dimension formula for $\mathcal{I}(k, d, n)$ as a positive sum involving symmetric powers of $V_{Q}$. The two formulas are related by an identity involving binomial coefficients. There is some superficial similarity to equations involved in the binomial transform of combinatorics, but the identity at hand is different. As we will explain, the key identity which makes it work is a property of the Tricomi Confluent Hypergeometric Function. Consequently, we name it the Confluent Binomial transform (CBT). In this section we will develop these ideas discussing the dimension formula for $\mathcal{I}(k, d, n)$ as a positive sum in detail. This forms the foundation for a construction algorithm for the LWPs. For this reason, we will refer to the positive dimension formula in terms of a positive sum as the construction formula. To go beyond counting and get the construction algorithm for the LWPs requires a discussion of an inner product.

\subsection{From resolution to construction: counting without signs}

Using characters we have obtained the generating function of the number of LWPs as follows

$$
\frac{\left(1-s^{2}\right)^{n}}{(1-s)^{d(n-1)}}=\sum_{l=0}^{\infty} L(l, d, n) s^{l}
$$

We will derive an interesting expression for $L(l, d, n)$ in terms of $S(k, d, n)$ which is the dimension of $\operatorname{Sym}^{k}\left(V_{d H}\right)$. Our starting point is the explicit expression

$$
S(k, d, n)=\frac{((n-1) d+k-1) !}{k !((n-1) d-1) !} \equiv S(k, D=d(n-1))
$$

The second equality emphasizes the fact that this depends only on $D=d(n-1)$. Observe that

$$
\frac{1}{(1-s)^{D}}=\sum_{k=0}^{\infty} S(k, D) s^{k}
$$

or

$$
\frac{1}{(1-s)^{d(n-1)}}=\sum_{k=0}^{\infty} S(k, d, n) s^{k}
$$

Then we have

$$
\begin{aligned}
\frac{\left(1-s^{2}\right)^{n}}{(1-s)^{d(n-1)}} & =\sum_{p=0}^{\infty} \sum_{k=0}^{n} S(p, d, n) \frac{n !(-1)^{k}}{k !(n-k) !} s^{p+2 k} \\
& =\sum_{l=0}^{\infty} \sum_{k=0}^{n} S(l-2 k, d, n) \frac{n !(-1)^{k}}{k !(n-k) !} s^{l}
\end{aligned}
$$

We now find

$$
L(l, d, n)=\sum_{k=0}^{n} \frac{(-1)^{k} n !}{k !(n-k) !} S(l-2 k, d, n)
$$


which is precisely the dimension formula that is implied by the exact sequences described in section 4. We will now argue that there is a second formula relating $L(l, d, n)$ and $S(l-2 k, d, n)$, given by

$$
S(p, d, n)=\sum_{i=0}^{\left\lfloor\frac{p}{2}\right\rfloor} L(p-2 i, d, n) \frac{(n+i-1) !}{i !(n-1) !}
$$

Start by substituting the formula (6.6) for $L(k, d, n)$ in terms of $S(k, d, n)$ into (6.7) to find

$$
\begin{aligned}
S(p, d, n) & =\sum_{i=0}^{\left\lfloor\frac{p}{2}\right\rfloor} \sum_{k=0}^{n} \frac{(-1)^{k} n !}{k !(n-k) !} \frac{(n+i-1) !}{i !(n-1) !} S(p-2 i-2 k, d, n) \\
& =\sum_{m=0}^{\left\lfloor\frac{p}{2}\right\rfloor} \sum_{k=0}^{\min (m, n)} \frac{(-1)^{k} n}{(m-k) ! k !(n-k) !} S(p-2 m, d, n)
\end{aligned}
$$

This is indeed an equality, which follows after using the identity

$$
\sum_{k=0}^{\min (m, n)} \frac{(-1)^{k}}{(m-k) ! k !(n-k) !}=\frac{1}{n} \delta_{m, 0}
$$

The equation (6.7) implies, after subtracting the $i=0$ term $L(p, d, n)$, that the dimension of the ideal generated by $V_{Q}$ is

$$
\operatorname{Dim}(\mathcal{I}(p, d, n))=\sum_{i=1}^{\left\lfloor\frac{p}{2}\right\rfloor} L(p-2 i, d, n) \frac{(n+i-1) !}{i !(n-1) !}
$$

It turns out that the identity (6.9) is related to a hypergeometric function. Introduce the function

$$
F(x ; m, n)=\sum_{m=0}^{\left\lfloor\frac{p}{2}\right\rfloor} \sum_{k=0}^{\min (m, n)} \frac{(x)^{k}}{(m-k) ! k !(n-k) !}
$$

With the help of Mathematica, we find

$$
F(x ; m, n)=\frac{(-1)^{m} x^{m}}{m ! n !} U\left[-m, 1-m+n,-x^{-1}\right]
$$

where $U$ is a tricomi confluent hypergeometric function. Consequently we have

$$
\sum_{m=0}^{\left\lfloor\frac{p}{2}\right\rfloor} \sum_{k=0}^{\min (m, n)} \frac{(x)^{k}}{(m-k) ! k !(n-k) !}=\frac{(-1)^{m} x^{m}}{m ! n !} U\left[-m, 1-m+n,-x^{-1}\right]
$$

This reduces our identity to a property of the tricomi confluent hypergeometric function when the last argument is 1

$$
\frac{1}{m ! n !} U[-m, 1-m+n, 1]=\frac{1}{n} \delta_{m, 0}
$$

The equation (6.10) gives the dimension of the ideal at each $k$, as a sum of positive terms. The ideal generated by the quadratic polynomials $\left\{Q_{A}: 0 \leq A \leq n-1\right\}$ consists of 
expressions of the form $\sum_{A} h_{A} Q_{A}$ where $h_{A} \in \mathcal{R}=\mathbb{C}\left[X_{\mu}^{A}\right]$. We can organize the ideal, as a vector space over $\mathbb{C}$, according to how many $Q$ 's they contain if we restrict the coefficients $h_{A}$ to be without $Q$ 's, in other words to belong to the quotient space $\mathcal{R} / \mathcal{I}$. Elements of degree $k$ containing a single $Q$, for example, are of the form

$$
\sum_{A} l_{A} Q_{A}
$$

where $l_{A}$ is in $\mathcal{L}(k-2, d, n)$, the space of LWPs of degree $k-2$. Consequently, a subspace of $\mathcal{I}(k, d, n)$ is

$$
\mathcal{L}(k-2, d, n) \otimes V_{Q}
$$

At this point, we use the identity (6.7), derived with the help of the confluent binomial transform, to decompose $\mathcal{I}(k, d, n)$ as

$$
\mathcal{I}(k, d, n)=\bigoplus_{i=1}^{\left\lfloor\frac{k}{2}\right\rfloor} \mathcal{L}(k-2 i, d, n) \otimes \operatorname{Sym}^{i}\left(V_{Q}\right)
$$

This decomposition gives a way of constructing the space of LWPs, recursively in $k$. Start with $k=2$.

$$
\operatorname{Sym}^{2}\left(V_{d H}\right)=V_{Q} \oplus \mathcal{L}(2, d, n)
$$

From this we read off the fact that the ideal $\mathcal{I}(2, d, n)$ is $V_{Q}$ and $\mathcal{L}(2, d, n)$ is the complement to $V_{Q}$. With an appropriate inner product, to be discussed in the next section, this will be an orthogonal complement. Now use (6.17) to write

$$
\mathcal{I}(4, d, n)=\left(\mathcal{L}(2, d, n) \otimes V_{Q}\right) \oplus \operatorname{Sym}^{2}\left(V_{Q}\right)
$$

We know $\mathcal{L}(2, d, n)$ from the first step so we can construct this. We then we take the orthogonal complement to $\mathcal{I}(4, d, n)$ in $\operatorname{Sym}^{4}\left(V_{d H}\right)$ to obtain $\mathcal{L}(4, d, n)$. We then repeat the process: from (6.17) we can construct $\mathcal{I}(6, d, n)$ using

$$
\mathcal{I}(6, d, n)=\left(\mathcal{L}(4, d, n) \otimes V_{Q}\right) \oplus\left(\mathcal{L}(2, d, n) \otimes \operatorname{Sym}^{2}\left(V_{Q}\right) \oplus \operatorname{Sym}^{3}\left(V_{Q}\right)\right)
$$

Now take the orthogonal complement to $\mathcal{I}(6, d, n)$ in $\operatorname{Sym}^{6}\left(V_{d H}\right)$ and we get $\mathcal{L}(6, d, n)$. A similar construction starting from $\mathcal{L}(1, d, n)=V_{d H}$ will give a recursive construction for all the odd $k$ cases.

Much as the exact sequences of vector spaces over $\mathbb{C}$ are related to exact sequences of modules over $\mathcal{R}=\mathbb{C}\left[X_{\mu}^{A}\right]$, the above equations decomposing the polynomials in $X_{\mu}^{A}$ of each degree $k$, can be collected into a statement about the ring $\mathcal{R}$. The quadratic $Q_{A}$ 's span the vector space $V_{Q}$. The symmetric algebra of $V_{Q}$, denoted by $\operatorname{Sym}\left(V_{Q}\right)$ is the direct sum of symmetrised tensor products of all degrees

$$
\operatorname{Sym}\left(V_{Q}\right)=\bigoplus_{k=0}^{\infty} \operatorname{Sym}_{\mathbb{C}}^{k}\left(V_{Q}\right)
$$

The degree 0 part is defined as $\mathbb{C}$. The above decompositions of $\mathcal{R}$ at each degree are captured by

$$
\mathcal{R}(d, n)=\mathcal{R}(d, n) / \mathcal{I}(d, n) \otimes_{\mathbb{C}} \operatorname{Sym}_{\mathbb{C}}\left(V_{Q}\right)
$$


which indeed follows from the fact that $\mathcal{I}$ is generated by quadratic constraints spanning $V_{Q}$. The subscript $\mathbb{C}$ indicates that we are tensoring over the base field, not over the ring.

\subsection{Implementing the construction using the natural inner product}

In the last section we have described an algorithm for the construction of the polynomials that correspond to primary operators. The algorithm works by recursively proceeding in degree, using orthogonality to construct the higher degree spaces from the lower degree ones. The only missing ingredient in the algorithm was the question of which inner product should be used. In this section we will fill this hole and give a detailed account of the algorithms that have been implemented using Mathematica.

Recall that the LWPs are both translation invariant and harmonic. The requirement of translation invariance is the primary constraint, written using the differential operator realization of the conformal group which sets $K_{\mu}=\frac{\partial}{\partial x^{\mu}}$. The condition that the polynomials are harmonic follows from the equation of motion for the free scalar. The irrep $V_{+}$ corresponding to states of the free scalar field, is the space spanned by harmonic and translation invariant polynomials in $x_{\mu}$. To deal with polynomials that correspond to primaries built using a product of $n$ scalar fields, we replace $x_{\mu} \rightarrow x_{\mu}^{I}$ with $I=1,2, \cdots, n$. The LWPs are now given by the solution to a system of differential equations involving COM conditions ( $d$ equations) and the Laplacian conditions ( $n$ equations).

Our construction makes use of a natural inner product on polynomials in $x_{\mu}$, defined by

$$
<x_{\mu_{1}} \cdots x_{\mu_{k}}, x_{\nu_{1}} \cdots x_{\nu_{k}}>=\frac{1}{k !} \sum_{\sigma \in S_{k}} \delta_{\mu_{1}, \nu_{\sigma(1)}} \delta_{\mu_{2}, \nu_{\sigma(2)}} \cdots \delta_{\mu_{k}, \nu_{\sigma(k)}}
$$

Polynomials of different degree are orthogonal. There is an obvious extension to polynomials in the multi-particle system, i.e. polynomials of degree $k$ in $x_{\mu}^{I}$ as follows

$$
<x_{\mu_{1}}^{I_{1}} \cdots x_{\mu_{k}}^{I_{k}}, x_{\nu_{1}}^{J_{1}} \cdots x_{\nu_{k}}^{J_{k}}>=\frac{1}{k !} \sum_{\sigma \in S_{k}} \delta_{\mu_{1}, \nu_{\sigma(1)}} \delta^{I_{1} J_{\sigma(1)} \cdots \delta_{\mu_{k}, \nu_{\sigma(k)}}} \delta^{I_{k} J_{\sigma(k)}}
$$

The construction starts by recognizing that harmonic polynomials can be obtained as an orthogonal subspace, with orthogonality given by the above inner product. To make the argument, start by noting that polynomials of a fixed degree $k$ in $x_{\mu}$ correspond to symmetric tensors of degree $k$. The symmetric tensors span the space

$$
\operatorname{Sym}^{k}\left(V_{d}\right)
$$

We will now argue that symmetric tensors with non-vanishing trace form a vector subspace of $\operatorname{Sym}^{k}\left(V_{d}\right)$ which is orthogonal to the traceless tensors. The traceless tensors correspond to harmonic polynomials, which establishes the result.

For simplicity, start with the single particle case. Consider the differential operator $\sum_{\mu, \nu=1}^{d} x_{\mu} x_{\mu} \frac{\partial^{2}}{\partial x_{\nu} \partial x_{\nu}}$. Let it act on all polynomials of fixed degree - it is a linear operator on this space. This linear operator is hermitian with respect to the natural inner product introduced above and hence it is diagonalisable. The harmonic polynomials are the null eigenvectors, while the traceful eigenvectors belong to the non-zero eigenspace. Since the 
linear operator is hermitian eigenstates of different eigenvalue are orthogonal with respect to the inner product introduced above. This proves that, for the single particle case, the traceless tensors form an orthogonal subspace of $\operatorname{Sym}^{k}\left(V_{d}\right)$.

The single particle argument is easily generalized: the polynomials in $x_{\mu}^{I}$ which are annihilated by all $n$ Laplacians must be orthogonal to any polynomial that is not annihilated by one or more Laplacians. The hermitian linear operator that plays a role in the multi-particle case is the sum of the single particle operators

$$
\mathcal{O}_{\mathcal{L}} \equiv \sum_{I=1}^{n} \sum_{\mu, \nu=1}^{d} x_{\mu}^{I} x_{\mu}^{I} \frac{\partial^{2}}{\partial x_{\nu}^{I} \partial x_{\nu}^{I}}
$$

Anything harmonic in all the $x_{\mu}^{I}$ is in the null space of $\mathcal{O}_{\mathcal{L}}$. Any eigenvector not annihilated by all the Laplacians belongs to a non-zero eigenspace of $\mathcal{O}_{\mathcal{L}}$. Since eigenfunctions with distinct eigenvalues are orthogonal this proves that the multi-harmonic polynomials are orthogonal to the polynomials which are not multi-harmonic.

We still need to consider the $K_{\mu}$ conditions which we have named the COM conditions above. A polynomial of degree $k$ in the $x_{\mu}^{I}$ is an element of $\operatorname{Sym}^{k}\left(V_{d} \otimes V_{\text {nat }}\right)$. We will first outline the argument for the simplest case of $d=1$. Start from the observation that $\operatorname{Sym}^{k}\left(V_{\text {nat }}\right)=\operatorname{Sym}^{k}\left(V_{0} \oplus V_{H}\right)$ which implies the decomposition

$$
\operatorname{Sym}^{k}\left(V_{\text {nat }}\right)=\operatorname{Sym}^{k}\left(V_{0} \oplus V_{H}\right)=\sum_{l=0}^{k} \operatorname{Sym}^{l}\left(V_{0}\right) \otimes \operatorname{Sym}^{(k-l)}\left(V_{H}\right)
$$

Since the natural inner product is $S_{n}$ invariant, this decomposition is orthogonal with respect the natural inner product

$$
<x^{I}, x^{J}>=\delta^{I J}
$$

The $l=0$ subspace is annihilated by the COM differential operator. Thus, polynomials that obey the center of mass condition again form an orthogonal subspace of $\operatorname{Sym}^{k}\left(V_{1}\right)$. A second approach to demonstrate the same fact, makes use of the operator

$$
\mathcal{O}_{1}=\sum_{I, J=1}^{n} x^{I} \frac{\partial}{\partial x^{J}}
$$

which is hermitian with respect to the natural inner product. The translation invariant polynomials belong to the null space of $\mathcal{O}_{1}$, while the non-translation invariant eigenvectors are not annihilated by $\mathcal{O}_{1}$ and hence belong to a non-zero eigenspace of $\mathcal{O}_{1}$. Since eigenfunctions of a hermitian operator with distinct eigenvalues are orthogonal we arrive at our earlier conclusion that polynomials obeying the center of mass condition again form an orthogonal subspace of $\operatorname{Sym}^{k}\left(V_{1}\right)$. As for the Laplacian discussion above, we can generalize this discussion from $d=1$ to general $d$. For general $d$ we consider

$$
\mathcal{O}_{c m}=\sum_{I, J=1}^{n} \sum_{\mu=1}^{d} x_{\mu}^{I} \frac{\partial}{\partial x_{\mu}^{J}}
$$


The null space of $\mathcal{O}_{c m}$ are polynomials invariant under simultaneous translation $x_{\mu}^{I} \rightarrow$ $x_{\mu}^{I}+a_{\mu}$. Anything not annihilated by $\mathcal{O}_{c m}$ belongs to a non-zero eigenspace of $\mathcal{O}_{c m}$. Since eigenfunctions with distinct eigenvalues are orthogonal this proves that the translation invariant polynomials are an orthogonal subspace of $\operatorname{Sym}^{k}\left(V_{d} \otimes V_{\text {nat }}\right)$ for any $d$.

We are now ready to describe our construction algorithm. The space of polynomials of fixed degree can be decomposed, with respect to the hermitian operators $\mathcal{O}_{c m}$ and $\mathcal{O}_{\mathcal{L}}$ in terms of null and positive eigenvalues as follows

$$
\begin{aligned}
& \mathcal{R}(k)=(\mathcal{R}(k))_{0}^{c m} \oplus(\mathcal{R}(k))_{+}^{c m} \\
& \mathcal{R}(k)=(\mathcal{R}(k))_{0}^{\mathcal{L}} \oplus(\mathcal{R}(k))_{+}^{\mathcal{L}}
\end{aligned}
$$

This decomposition is orthogonal with respect to the natural inner product. The LWPs are in

$$
(\mathcal{R}(k))_{0}^{c m} \cap(\mathcal{R}(k))_{0}^{\mathcal{L}}
$$

In the form just described, it is straight forward to produce a Mathematica implementation of the construction algorithm that gives the full set of LWPs. Run times increase as the degree $k$ is increased.

An alternative construction algorithm that exploits more of the $S_{n}$ structure of the problem, starts by considering polynomials in the hook variables $X_{\mu}^{A}, A=1, \cdots, n-1$. The advantage is that these polynomials already satisfy the COM condition, so we only need to impose (2.42) and (2.43). Again, by using Mathematica to pick up the subspace orthogonal to $(2.42)$ and (2.43) we have also implemented this alternative construction algorithm. The above discussion implies that the space of degree $k$ polynomials can be decomposed as

$$
p^{(k)}=p_{h}^{(k)}+Q_{A} p_{h, A}^{(k-2)}+Q_{A} Q_{B} p_{h, A B}^{(k-4)}+\cdots
$$

$p_{h}^{(k)}, p_{h, A}^{(k)}, \cdots$ are all polynomials of degree $k$ which are annihilated by the Laplacians $\square_{A}$ defined in (2.49). In the expansion only the first term $p_{h}^{(k)}$ is in $(\mathcal{R}(k))_{0}^{\mathcal{L}}$. The remaining terms belong to $(\mathcal{R}(k))_{+}^{\mathcal{L}}$. In the expansion of $p^{(k)}$, the leading term $p_{h}^{(k)}$ is orthogonal to all of the subsequent terms.

\subsection{Commutative star product on lowest weight polynomials}

As we saw in section 2 the lowest weight polynomials are in 1-1 correspondence with a quotient ring, which has an associative product inherited from the quotient construction. Since the Laplacian constraints obeyed by the polynomials of the ring are second order differential operators, given two polynomials that obey the Laplacian constraints, the product of the two will, in general, fail to. Using (6.33) we will show there exists a suitable commutative star product so that given two polynomials that obey the Laplacian constraints, the star product of the two also obeys the constraints.

The components in the decomposition (6.33) can be organized by the grading defined by counting the number of $Q \mathrm{~s}$. $Q_{A} p_{h, A}^{(k-2)}$ is degree $1, Q_{A} Q_{B} p_{h, A B}^{(k-4)}$ degree 2 and so on. Polynomials obeying the Laplacian constraints are degree zero. The key idea behind the star product is that the degree just defined is additive: if polynomial $f_{1}$ is of degree $k_{1}$ and 
$f_{2}$ is of degree $k_{2}$ then the product $f_{1} f_{2}$ is of degree $k$ with $k \geq k_{1}+k_{2}$. This is almost obvious: consider the product of a degree $k_{1}$ and degree $k_{2}$ term

$$
Q_{A_{1}} \cdots Q_{A_{k_{1}}} p_{h, A_{1} \cdots A_{k_{1}}}^{\left(q_{1}\right)} Q_{B_{1}} \cdots Q_{B_{k_{2}}} p_{h, B_{1} \cdots B_{k_{2}}}^{\left(q_{2}\right)}
$$

As we explained above, the product $p_{h, A_{1} \cdots A_{k_{1}}}^{\left(q_{1}\right)} p_{h, B_{1} \cdots B_{k_{2}}}^{\left(q_{2}\right)}$ will not in general obey the Laplacian constraints. Consequently we can again use the decomposition (6.33) to write

$$
p_{h, A_{1} \cdots A_{k_{1}}}^{\left(q_{1}\right)} p_{h, B_{1} \cdots B_{k_{2}}}^{\left(q_{2}\right)}=p_{h, A_{1} \cdots A_{k_{1}} B_{1} \cdots B_{k_{2}}}^{\left(q_{1}+q_{2}\right)}+Q_{C} p_{h, A_{1} \cdots A_{k_{1}} B_{1} \cdots B_{k_{2}} C}^{\left(q_{1}+q_{2}-2\right)}+\cdots
$$

Inserting this back into (6.34) proves the result.

With this observation, we can define the star product. Using (6.33) decompose the product of two polynomials, which each obey the Laplacian constraints

$$
\begin{aligned}
f_{h}^{\left(k_{1}\right)} g_{h}^{\left(k_{2}\right)}= & (f g)_{h}^{\left(k_{1}+k_{2}\right)}+Q_{C}(f g)_{h, C}^{k_{1}+k_{2}-2}+\cdots \\
& +Q_{C_{1}} \cdots Q_{C_{m}}(f g)_{C_{1} \cdots C_{m}}^{\left(k_{1}+k_{2}-2 m\right)}
\end{aligned}
$$

The star product we want is

$$
f_{h}^{\left(k_{1}\right)} * g_{h}^{\left(k_{2}\right)}=(f g)_{h}^{\left(k_{1}+k_{2}\right)}
$$

Only the degree zero term survives because all higher degree terms are set to zero by the ideal of the ring.

We will now argue that this star product is associative. Recall that the usual product on polynomials is associative

$$
f_{h}^{\left(k_{1}\right)}\left(g_{h}^{\left(k_{2}\right)} h_{h}^{\left(k_{3}\right)}\right)=\left(f_{h}^{\left(k_{1}\right)} g_{h}^{\left(k_{2}\right)}\right) h_{h}^{\left(k_{3}\right)}
$$

Refining both sides of this last equation according to degree, we can write this as

$$
\begin{aligned}
& f_{h}^{\left(k_{1}\right)}\left((g h)_{h}^{\left(k_{2}+k_{3}\right)}+Q_{A}(g h)_{h, A}^{\left(k_{2}+k_{3}-2\right)}+\ldots\right) \\
& \quad=\left((f g)_{h}^{\left(k_{1}+k_{2}\right)}+Q_{A}(f g)_{h, A}^{\left(k_{1}+k_{2}-2\right)}+\ldots\right) h_{h}^{\left(k_{3}\right)} \\
& \left(f(g h)_{h}^{\left(k_{2}+k_{3}\right)}\right)_{h}^{\left(k_{1}+k_{2}+k_{3}\right)}+Q_{A}\left(f(g h)_{h}^{\left(k_{2}+k_{3}\right)}\right)_{h, A}^{\left(k_{1}+k_{2}+k_{3}-2\right)}+\ldots \\
& \quad=\left((f g)_{h}^{\left(k_{1}+k_{2}\right)} h\right)_{h}^{\left(k_{1}+k_{2}+k_{3}\right)}+Q_{A}\left((f g)_{h}^{\left(k_{1}+k_{2}\right)} h\right)_{h, A}^{\left(k_{1}+k_{2}+k_{3}-2\right)}+\ldots
\end{aligned}
$$

Equating degree zero pieces on the two sides we have

$$
\left(f(g h)_{h}^{\left(k_{2}+k_{3}\right)}\right)_{h}^{\left(k_{1}+k_{2}+k_{3}\right)}=\left((f g)_{h}^{\left(k_{1}+k_{2}\right)} h\right)_{h}^{\left(k_{1}+k_{2}+k_{3}\right)}
$$

From our definition of the star product we have

$$
\begin{aligned}
& f *(g * h)=f *(g h)_{h}^{\left(k_{2}+k_{3}\right)}=\left(f(g h)_{h}^{\left(k_{2}+k_{3}\right)}\right)_{h}^{\left(k_{1}+k_{2}+k_{3}\right)} \\
& (f * g) * h=(f g)_{h}^{\left(k_{1}+k_{2}\right)} * h=\left((f g)_{h}^{\left(k_{1}+k_{2}\right)} h\right)_{h}^{\left(k_{1}+k_{2}+k_{3}\right)}
\end{aligned}
$$

It is now evident that

$$
f *(g * h)=(f * g) * h
$$

demonstrating that the star product is indeed associative.

It is reasonable to expect (based on the study of special instances of $n, d$ ) that this associative product can be expressed in terms of the ordinary product $f g$, corrected by products of the form $\mathcal{O}(f) \tilde{\mathcal{O}}(g)$ where $\mathcal{O}, \tilde{\mathcal{O}}$ are appropriate differential operators. Finding the explicit form of these operators in generality would be an interesting exercise for the future. 


\section{Further construction methods for lowest weight polynomials}

The construction algorithm we gave in the previous section has a recursive nature, and produces all the LWPs of degree up to any chosen maximum $k$. At each $k$, it uses orthogonality to elements written in terms of the LWPs at lower $k$. A second, more direct, algorithm works at fixed $k$, and implements the differential equations defining LWPs. A third algorithm works with projectors, and is based on analogies between the construction of LWPs and that of constructing symmetric traceless tensors. All of these algorithms have been tested in Mathematica. We give a brief discussion of algebraic geometry methods for constructing the quotient ring at hand.

\subsection{Intersection of kernels of two differential operators}

In this section we will outline a closely related but distinct construction algorithm. This new algorithm uses the fact that, as we explained earlier, the space of LWPs can be identified as the common null space of a set of differential operators. Here we will consider degree preserving version of the differential operators, and by using positive semi-definiteness properties, reduce the problem to that of finding the simultaneous null space of two differential operators. This last step is implemented in Mathematica.

Consider the space of polynomials of degree $k$ in the $d n$ variables $x_{\mu}^{I}$ where $1 \leq I \leq n$. The degree $k$ is a sum of degrees of $k=k_{1}+k_{2}+\cdots+k_{n}$, where $k_{I}$ is the degree in the $I$ 'th variable. A general polynomial with specified degrees $\left(k_{1}, k_{2}, \cdots, k_{n}\right)$ is

$$
\begin{aligned}
X_{\vec{\mu}}^{\vec{k}} & =X_{\mu_{11}, \mu_{12}, \cdots, \mu_{1 k_{1}} ; \mu_{21}, \mu_{22}, \cdots, \mu_{2 k_{2}} ; \cdots ; \mu_{n 1}, \mu_{n 2}, \cdots, \mu_{n k_{n}}} \\
& =x_{\mu_{11}}^{1} \cdots x_{\mu_{1 k_{1}}}^{1} x_{\mu_{21}}^{2} \cdots x_{\mu_{2 k_{2}}}^{2} \cdots x_{\mu_{n 1}}^{n} \cdots x_{\mu_{n k_{n}}}^{n}
\end{aligned}
$$

All the $\mu$ indices take values in the range $1 \leq \mu \leq d . X$ is symmetric in the first $k_{1}$ indices, the next $k_{2}$ indices, etc. The number of independent $X$ 's is

$$
\prod_{I=1}^{n} \frac{\left(d+k_{I}-1\right) !}{k_{I} !(d-1) !}
$$

We will be considering the vector space of the $X$ 's, for all $\vec{k}$ satisfying $\sum_{I} k_{I}=k$. This is equivalently a sum over partitions of $k$ with up to $n$ parts (since some of the $k_{I}$ could be zero). This vector space, denoted $\mathcal{W}_{k ; n, d}$, has dimension

$$
\begin{aligned}
\operatorname{Dim}\left(\mathcal{W}_{k ; n, d}\right) & =\sum_{k_{1}, k_{2}, \cdots, k_{n}=0}^{k} \delta\left(k, k_{1}+k_{2}+\cdots+k_{n}\right) \prod_{I=1}^{n} \frac{\left(d+k_{I}-1\right) !}{k_{I} !(d-1) !} \\
\mathcal{W}_{k ; n, d} & =\bigoplus_{\substack{k_{1}, k_{2} \cdots, k_{n}=0 \\
\sum_{I} k_{I}=k}}^{k} \operatorname{Sym}^{k_{1}}\left(V_{d}\right) \otimes \operatorname{Sym}^{k_{2}}\left(V_{d}\right) \otimes \cdots \otimes \operatorname{Sym}^{k_{n}}\left(V_{d}\right)
\end{aligned}
$$

On this subspace we have the linear operators

$$
\mathcal{O}_{\mathcal{L}}^{(I)}=\left(x^{I}\right)^{2} \square_{(I)}=\sum_{\alpha, \beta=1}^{d} x_{\alpha}^{I} x_{\alpha}^{I} \frac{\partial}{\partial x_{\beta}^{I} \partial x_{\beta}^{I}}
$$

which are degree preserving versions of the Laplacians. 
Consider the sum of these $n$ operators

$$
\mathcal{O}_{\mathcal{L}}=\sum_{I=1}^{n} \mathcal{O}_{\mathcal{L}}^{(I)}
$$

The null space of this operator obeys all the Laplacian conditions. The different Laplacian operators are commuting operators and they are all positive semi-definite operators, i.e. they all have eigenvalues which are non-negative. Consequently, the vanishing of the sum guarantees the vanishing of the summands. Thus, polynomials in the null space of $\mathcal{O}_{\mathcal{L}}$ are harmonic.

For each $\alpha \in\{1,2, \cdots, d\}$, we have a centre of mass operator

$$
\frac{\partial}{\partial x_{\alpha}^{C M}}=\sum_{I=1}^{n} \frac{\partial}{\partial x_{\alpha}^{I}}
$$

Polynomial functions of $\left\{x_{\alpha}^{I}: 1 \leq I \leq n\right\}$ can be factored into sums of centre of massdependent functions times functions of the differences. The degree-preserving COM operator

$$
\sum_{\alpha} x_{\alpha}^{C M} \frac{\partial}{\partial x_{\alpha}^{C M}}=\sum_{\alpha} \sum_{I, J=1}^{n} x_{\alpha}^{I} \frac{\partial}{\partial x_{\alpha}^{J}}
$$

is positive semi-definite. Any operator of fixed degree, annihilated by $\frac{\partial}{\partial x_{\alpha}^{C M}}$ is also annihilated by $x_{\alpha}^{C M} \frac{\partial}{\partial x_{\alpha}^{C M}}$. Therefore the following sum

$$
\begin{aligned}
\mathcal{O}_{\mathcal{C M}} & =\sum_{\alpha=1}^{d} x_{\alpha}^{C M} \frac{\partial}{\partial x_{\alpha}^{C M}} \\
& =\sum_{I, J=1}^{n} \sum_{\alpha=1}^{d} x_{\alpha}^{I} \frac{\partial}{\partial x_{\alpha}^{J}}
\end{aligned}
$$

has the property that its null space is the simultaneous null space of all the $d$ COM operators.

The null space of $\mathcal{O}_{\mathcal{C M}}$ obeys the lowest weight condition. We build the combined operator

$$
\mathcal{O}=\left(\begin{array}{c}
\mathcal{O}_{\mathcal{L}} \\
\mathcal{O}_{\mathcal{C M}}
\end{array}\right)
$$

$\mathcal{O}$ is an operator in $\operatorname{End}\left(\mathcal{W}_{k ; n, d}\right)$. The null space of this operator is the space of lowest weight polynomials of degree $k$. We have implemented this algorithm in Mathematica and have checked that the dimension of the null space of $\mathcal{O}$ does indeed agree with the number of LWPs.

\subsection{Constraints and projectors on $V_{d H} \otimes V_{d H}$}

An interesting algebraic angle on the primaries problem is that in some sense it is a generalization of the problem of finding symmetric traceless tensors of $\mathrm{SO}(d)$. Nice bases for 
these tensors can be constructed using Young diagram techniques for $S O$ groups. These symmetric traceless tensors are annihilated by contraction operators. The contraction tensors form part of a Brauer algebra, so the symmetric traceless tensors of rank $k$ for $s o(d)$ are related to irreps of a Brauer algebra. This follows from the fact that the commutant of $\mathrm{SO}(d)$ in $V_{d}^{\otimes k}$, where $V_{d}$ is the fundamental of $s o(d)$, is the Brauer algebra. In the problem of symmetric tensors we are trying to find tensors

$$
T_{\mu_{1}, \mu_{2}, \cdots, \mu_{k}}
$$

such that

$$
T_{\mu, \mu, \mu_{3}, \cdots, \mu_{k}}=0
$$

Note that, because $T$ is symmetric, we can move the paired indices to any slot. Another way to phrase this is by considering the contraction operator

$$
C_{12} T_{\mu_{1}, \cdots, \mu_{k}}=\delta_{\mu_{1}, \mu_{2}} T_{\mu, \mu, \mu_{3}, \cdots, \mu_{k}}=0
$$

The contraction operator is a projector from $V_{d} \otimes V_{d}$ to the trivial rep of $s o(d)$.

In the present case, there is a natural generalized symmetric tensor in the game

$$
T_{\mu_{1}, \mu_{2}, \cdots, \mu_{k}}^{A_{1}, A_{2}, \cdots, A_{k}} \leftrightarrow X_{\mu_{1}}^{A_{1}} X_{\mu_{2}}^{A_{2}} \cdots X_{\mu_{k}}^{A_{k}}
$$

The symmetry is the $S_{k}$ group of permutations of the pairs $(\mu, A)$. To start it is constructive to consider the $k=2$ case. Define

$$
V_{d H}=V_{d} \otimes V_{H}
$$

We are looking at the subspace of

$$
V_{d H}^{\otimes 2}
$$

which is invariant under the $S_{2}$ permutation of the two factors, i.e. we are looking at

$$
\operatorname{Sym}^{2}\left(V_{d H}\right)
$$

Now $V_{d} \otimes V_{d}$ contains a symmetric rank 2 tensor, an anti-symmetric rank two tensor and a trace (invariant) of $\mathrm{SO}(d)$ all with multiplicity 1 . The product $V_{H} \otimes V_{H}$ decomposes under the diagonal $S_{n}$ as

$$
V_{H} \otimes V_{H}=V_{0} \oplus V_{H} \oplus V_{[n-2,2]} \oplus V_{[n-2,1,1]}
$$

The symmetric part contains the first three spaces in the direct sum

$$
\operatorname{Sym}^{2}\left(V_{H} \otimes V_{H}\right)=V_{0} \oplus V_{H} \oplus V_{[n-2,2]}
$$

The constraints tell us that the projection to $\left(V_{0}^{\left(S_{n}\right)} \oplus V_{H}^{\left(S_{n}\right)}\right) \otimes V_{0}^{\mathrm{SO}(d)}$ inside $\operatorname{Sym}^{2}\left(V_{d H}\right)$ vanishes. So we are looking at vectors

$$
v \in \operatorname{Sym}^{2}\left(V_{d H}\right)
$$

which obey

$$
\left(P_{0}^{(\mathrm{SO}(d))}\left(P_{0}^{\left(S_{n}\right)}+P_{H}^{\left(S_{n}\right)}\right)\right) \quad v=0
$$


Let us define

$$
P^{\mathcal{L}}=\left(P_{0}^{(\mathrm{SO}(d))}\left(P_{0}^{\left(S_{n}\right)}+P_{H}^{\left(S_{n}\right)}\right)\right)
$$

The operator $P^{\mathcal{L}}$ is a projector obeying $\left(P^{\mathcal{L}}\right)^{2}=P^{\mathcal{L}}$ which follows from

$$
\begin{aligned}
\left(P_{0}^{(\mathrm{SO}(d))}\right)^{2} & =P_{0}^{(\mathrm{SO}(d))} \\
\left(P_{0}^{\left(S_{n}\right)}\right)^{2} & =P_{0}^{\left(S_{n}\right)} \\
\left(P_{H}^{\left(S_{n}\right)}\right)^{2} & =P_{H}^{\left(S_{n}\right)} \\
P_{0}^{\left(S_{n}\right)} P_{H}^{\left(S_{n}\right)} & =0 \\
P_{0}^{(\mathrm{SO}(d))} P_{0}^{\left(S_{n}\right)} & =P_{0}^{\left(S_{n}\right)} P_{0}^{(\mathrm{SO}(d))} \\
P_{0}^{(\mathrm{SO}(d))} P_{H}^{\left(S_{n}\right)} & =P_{H}^{\left(S_{n}\right)} P_{0}^{(\mathrm{SO}(d))}
\end{aligned}
$$

This is a projector $P^{\mathcal{L}}$ which acts on pairwise slots. It is the analog of the contraction tensor of the Brauer algebra. Combine the $\mu, A$ indices into a composite index $M$. We are considering symmetric tensors

$$
T_{M_{1}, \cdots, M_{k}}
$$

that are annihilated by $P^{\mathcal{L}}$

$$
P_{12}^{\mathcal{L}} T_{M_{1}, M_{2}, \cdots, M_{k}}=P_{M_{1} M_{2}}^{N_{1}, N_{2}} T_{N_{1}, N_{2}, M_{3}, \cdots, M_{k}}=0
$$

We have a very concrete formula for $\kappa_{A B C}$ and hence for $P$

$$
(P)_{\mu_{1}, A_{1}, \mu_{2}, A_{2}}^{\nu_{1}, B_{1} ; \nu_{2}, B_{2}}=\sum_{A=0}^{n-1} \delta_{\mu_{1}, \mu_{2}} \delta_{\nu_{1}, \nu_{2}} \kappa_{A, A_{1}, A_{2}} \kappa_{A, B_{1}, B_{2}}
$$

The symmetry of $T$ then also implies

$$
P_{M_{1} M_{2}}^{N_{1}, N_{2}} T_{N_{1}, M_{3}, N_{2}, \cdots, M_{k}}=0
$$

and so on for any pair. We will now argue that to find a linear basis at degree $k$ in the ring of LWPs, we have to consider symmetric tensors $T$ obeying equation (7.25).

Use the inner product for polynomials in $x_{\mu}^{I}$ used before. This induces an inner product of the same form on $X_{\mu}^{A}$. For operator

$$
\square_{A}=\sum_{\mu=1}^{d} \sum_{B, C=1}^{n-1} \kappa_{A B C} \frac{\partial^{2}}{\partial X_{\mu}^{B} \partial X_{\mu}^{C}}
$$

consider

$$
\left(\square_{A}\right)^{\dagger} \square_{A}=\sum_{B, C, D, E=0}^{n-1} \sum_{\mu, \nu=1}^{d} X_{\nu}^{D} X_{\nu}^{E} \kappa_{A D E} \kappa_{A B C} \frac{\partial^{2}}{\partial X_{\mu}^{B} \partial X_{\mu}^{C}}
$$

This is a positive semi-definite operator. Any eigenvector $v$ of eigenvalue $\lambda$ has the property

$$
\left(v, \square_{A}^{\dagger} \square_{A} v\right)=\lambda(v, v)=\left(\square_{A} v, \square_{A} v\right) \geq 0
$$

so $\lambda \geq 0$. Hence being in the simultaneous null spaces is equivalent to being in the null space of

$$
\sum_{A=0}^{n-1}\left(\square_{A}\right)^{\dagger} \square_{A}
$$

Symmetric tensors in this null space are equivalently in the null space of $P_{12}^{\mathcal{L}}$. 


\subsection{Standard algebraic geometry methods for $\mathcal{R} / \mathcal{I}$}

As explained in section 2, the LWPs are in 1-1 correspondence with the elements of $\mathcal{R} / \mathcal{I}$. The quotient ring is defined in terms of equivalence classes. Each equivalence class contains an LWP. There are standard algebraic geometry methods, based on Groebner bases, for the construction of the equivalence classes. The Groebner bases rely on choosing certain orderings on monomials [6]. To pick the LWPs within each equivalence class would probably be a non-trivial additional step. The polynomials $Q_{A}$ are polynomials with integer coefficients, so another approach to the quotient ring may be to use an analog of Groebner bases which works for rings defined over integers (see e.g. [12]). If we are interested in the effective action problem [3], it is only the quotient ring which is of interest. If we are interested in constructing primary fields in CFT, the specific LWPs are important. It will be interesting to investigate the efficacy of the different algorithms given here, relative to the algebraic geometry methods, from the point of view of primary fields as well as from the point of view of effective actions.

\section{Discussion and future directions}

We have considered the problem of constructing primary fields in free scalar CFTs in general dimensions, combining insights from $[1,2,4]$ and [3]. This has been a fruitful avenue, with the key results described in the introduction and developed in the bulk of the paper.

A number of future projects are suggested by our results. We have given a number of Mathematica constructions of lowest weight polynomials. These codes are available upon request.

It would be interesting to compare the efficiency of the different algorithms discussed in sections 6 and 7. Extending the present work to fermions and gauge fields is a worthwhile avenue. For the holomorphic sector of primaries in the free fermion theory see [13].

To get primary fields in the CFT, we have to project to $S_{n}$ invariants. The Hilbert series of the $S_{n}$ invariants is easy to write in terms of those for the LWPs (see [4]). A complete description of the ring structure for $S_{n}$ invariants in $d=3,4$ is a worthwhile goal in the short term.

It would be interesting to investigate for more general rings, the connection between resolution and construction we have given in section 6 . In the present case all the constraints are quadratic. There should be closely related generalizations when the constraints are each homogeneous but of different degrees.

\subsection{Further developing the analogy to tracelessness: a generalization of Brauer algebras}

In section 7.2 we developed an approach to the construction of LWPs, based on projectors acting on degree $k$ polynomials in $X_{\mu}^{A}$. These polynomials form a vector space isomorphic to the space of symmetric tensors $\operatorname{Sym}^{k}\left(V_{d H}\right)$. It is useful to consider the tensor product $V_{d H}^{\otimes k}$ where we have the projectors $\mathcal{P}_{i j}^{\mathcal{L}}$ acting on the slots labeled $i, j$, and subsequently projecting to the $S_{k}$ symmetric part. This is analogous to the problem of constructing symmetric 
traceless tensors in $V_{d}^{\otimes k}$. In this case there are finite algebras, the Brauer algebras $D_{k}(d)$ the commutant of $o(d)$ in $V_{d}^{\otimes k}$, which give a representation theory meaning to this construction. For the representation theory of $D_{k}(d)$, see for example [14]. In the present case, we have an analog of the Brauer algebra, namely the algebra generated by $\mathcal{P}_{i j}^{\mathcal{L}} \subset \operatorname{End}\left(V_{d H}^{\otimes k}\right)$ along with permutations in $S_{k}$. Let us call this algebra $\mathcal{A}(k, d, n)$. It is plausible that the LWPs form irreducible reps of this algebra. It would be interesting to investigate this conjecture.

As explained in [4], part of the motivation for studying free field primaries comes from the goal of finding a uniform framework of algebraic structures, based on two dimensional topological field theory (TFT2), for understanding both the space-time dependence and the combinatoric structure of correlators in $N=4 \mathrm{SYM}$. The combinatorics of the half-BPS sector is controlled by a Frobenius algebra (TFT2) which is the space of conjugacy classes of symmetric groups $S_{n}$ for all $n$, resulting in useful Young diagram bases for AdS/CFT [15]. For the quarter BPS sector at zero coupling, we have an algebra which is a subspace of $\mathbb{C}\left(S_{n+m}\right)$ invariant under conjugation by $S_{n} \times S_{m}$ [16-18]. This algebra arises as a way to describe nice bases for the ring of polynomial gauge invariant functions of two matrices $Y, Z$, which are orthogonal under free field inner products [19, 20]. Analogous constructions based on Brauer algebras provide an alternative approach to these orthogonal bases [21]. Generalizing these constructions to matrix systems forming representations of general symmetry groups led to an initial foray into the problem of constructing primary fields [22, 23].

The theme of finite dimensional algebras controlling questions about infinite dimensional representations of the conformal group has been a recurring theme. Developing the algebraic approach to LWPs based on representations of $\mathcal{A}(k, d, n)$ would be a concrete manifestation of the unity between algebraic structure for combinatorics and space-time dependence of correlators of gauge invariant observables.

\subsection{Quadratic algebras and Koszul algebras}

We have shown that the LWPs are in 1-1 correspondence with the quotient ring $\mathcal{R} / \mathcal{I}$, where $\mathcal{R}=\mathbb{C}\left[X_{\mu}^{A}\right]$ and $\mathcal{I}$ is generated by (2.44). This is an example of a quadratic algebra. These are defined by quotients of the tensor algebra $T(\mathbb{V})$ of a vector space $\mathbb{V}$, determined by a quadratic form $R \subset \mathbb{V} \otimes \mathbb{V}[24]$. In the present case, $\mathbb{V}=V_{d H}$ (let us use basis vectors $\left.e_{\mu}^{A}\right)$ and $R$ is spanned by

$$
\begin{gathered}
e_{\mu}^{A} \otimes e_{\nu}^{B}-e_{\nu}^{B} \otimes e_{\mu}^{A}, \\
\sum_{\mu} \sum_{B, C} \kappa_{A B C} e_{\mu}^{B} \otimes e_{\mu}^{C}
\end{gathered}
$$

The quotient is $T(\mathbb{V}) /<R>$. For the explicit definition of $<R>$ see Chapter 4 of [9]. It involves tensoring with arbitrary tensor powers of $\mathbb{V}$ on the left and right. The first line above ensures that we project from the tensor algebra to the symmetric algebra. Equivalently we go from generators of free algebras to commuting generators $X_{\mu}^{A}$. Every quadratic algebra has a dual quadratic algebra defined by $R^{\perp} \subset V^{*} \otimes V^{*}$. In the present case, we can work out that

$$
R=\Lambda^{2}\left(V_{d H}\right) \quad \oplus \quad \operatorname{Invt}_{s o(d)}\left(\operatorname{Sym}^{2}\left(V_{d}\right)\right) \otimes\left(\operatorname{Sym}^{2}\left(V_{H}\right)\right)_{[n]+[n-1,1]}
$$




$$
\begin{aligned}
& R^{\perp}=\Lambda^{2}\left(V_{d}^{*}\right)\left.\otimes \Lambda^{2}\left(V_{H}^{*}\right) \oplus \quad \operatorname{Sym}^{2}\left(V_{d}^{*}\right)\right)^{\prime} \otimes \operatorname{Sym}^{2}\left(V_{H}^{*}\right) \\
& \oplus \quad\left(\operatorname{Invt}\left(\operatorname{Sym}^{2}\left(V_{d}^{*}\right)\right) \otimes\left(\operatorname{Sym}^{2}\left(V_{H}^{*}\right)\right)_{[n-2,2]}\right.
\end{aligned}
$$

The details are not important. An important observation is that $T(\mathbb{V}) /<R>$ is a commutative algebra due to the presence of $\Lambda^{2}\left(V_{d H}\right)$ as a direct summand in $R$, while $T(\mathbb{V}) /<R^{\perp}>$ is not commutative due to the lack of such a direct summand.

A special class of quadratic algebras are said to be Koszul, which happens when the algebras form part of certain exact resolutions of the base field (see [9, 24]). Koszul algebras have a property of Koszul duality whereby the quadratic algebra and its dual quadratic algebra have equivalent derived categories of modules ( see [25] and references therein). An interesting question is whether $\mathcal{R} / \mathcal{I}$ is Koszul. If it is, this will be much more than a mathematical curiosity. It is plausible that modules of $\mathcal{R} / \mathcal{I}$ control the properties of primary fields for fermions, gauge fields and higher spin fields. This is expected by analogy to non-commutative geometry where the configuration space of a scalar field becomes a noncommutative algebra and the configuration spaces of non-trivial fields becomes modules over the algebra [26]. Thus if the Koszul property holds in the present case, the physics of $\mathcal{R} / \mathcal{I}$ may be equivalent to the physics of the Koszul dual algebra. This would indicate there might exist a hidden non-commutative reformulation of ordinary quantum field theory!

So is $\mathcal{R} / \mathcal{I}$ a Koszul algebra? Deformations of this algebra of lowest weight primaries, where the coefficients $\kappa_{A B C}$ are modified so that they satisfy a genericity condition, are Koszul [27]. A useful fact ( Example 2 following Corollary 6.3 in [24]) is that $\mathbb{C}\left[x_{1}, \cdots, x_{n}\right] /\left\langle q_{1}, \cdots, q_{r}\right\rangle$ is Koszul if $q_{1}, q_{2}, \cdots, q_{r}$ form a regular sequence of quadrics. Applying this to our case, the question is whether the $\left\{Q_{0}, Q_{1}, \cdots, Q_{n-1}\right\}$ (without deformation to reach the genericity condition of [27]) form a regular sequence. We leave this as a question for the future.

\subsection{Future direction: coherence relations between two products}

We showed in [4] that the OPE in free scalar theory can be used to define a commutative so $(4,2)$ covariant algebra with a non-degenerate bilinear pairing. The crossing equation of CFT becomes ordinary associativity of the algebra. Here we have seen that there is an algebra controlling primary fields for every $n$. The interplay between the commutative algebra coming from the OPE and the algebra studied here is an interesting question for the future.

\section{Acknowledgments}

This work of RdMK is supported by the South African Research Chairs Initiative of the Department of Science and Technology and National Research Foundation as well as funds received from the National Institute for Theoretical Physics (NITheP). SR is supported by the STFC consolidated grant ST/L000415/1 "String Theory, Gauge Theory \& Duality" and a Visiting Professorship at the University of the Witwatersrand, funded by a Simons Foundation grant held at the Mandelstam Institute for Theoretical Physics. SR thanks the Galileo Galilei Institute for Theoretical Physics for hospitality and the INFN for partial support during the completion of this work. 


\section{A The invariant in $V_{H} \otimes V_{H} \otimes V_{H}$}

In this section we will give the derivation of (2.32). Inserting the explicit expressions for the $S_{A I}$ we have

$$
\begin{aligned}
\sum_{I} S_{C I} S_{B I} S_{A I}= & \mathcal{N}_{A} \mathcal{N}_{B} \mathcal{N}_{C} \sum_{I}\left(-C \delta_{I, C+1}+\sum_{J_{1}=1}^{C} \delta_{J_{1}, I}\right) \\
& \times\left(-B \delta_{I, B+1}+\sum_{J_{2}=1}^{B} \delta_{J_{2}, I}\right)\left(-A \delta_{I, A+1}+\sum_{J_{3}=1}^{A} \delta_{J_{3}, I}\right)
\end{aligned}
$$

Expanding the brackets out there are 8 terms. Call them $T_{1}, T_{2}, \cdots T_{8}$. We will deal with each term separately in what follows.

$$
\begin{aligned}
T_{1} & =-A B C \sum_{I} \delta_{I, C+1} \delta_{I, B+1} \delta_{I, A+1} \\
& =-A B C \delta_{A B} \delta_{B C} \\
& =-A B C \delta_{A, B, C} \\
T_{2} & =B C \sum_{I=1}^{n} \delta_{I, C+1} \delta_{I, B+1} \sum_{J_{3}=1}^{A} \delta_{J_{3}, I} \\
& =B C \delta_{B, C} \sum_{I=1}^{n} \delta_{I, B+1} \sum_{J=1}^{A} \delta_{J, I} \\
& =B C \delta_{B, C} \sum_{I=1}^{A} \delta_{I, B+1} \\
& =B C \delta_{B, C} \Theta(B<A) \\
T_{3} & =A C \sum_{I} \delta_{I, C+1} \delta_{I, A+1} \sum_{J_{2}=1}^{B} \delta_{J_{2}, I} \\
& =A C \delta_{A, C} \sum_{I=1}^{n} \delta_{I, C+1} \sum_{J_{2}=1}^{B} \delta_{J_{2}, I} \\
& =A C \delta_{A, C} \sum_{I=1}^{B-1} \delta_{I, B-C}
\end{aligned}
$$

The last delta function is only non-zero if $B \geq C+1$. Define

$$
\begin{aligned}
\Theta(B>C) & =1 & & \text { if } B>C \\
& =0 & & \text { otherwise }
\end{aligned}
$$

We can then write

$$
T_{3}=A C \delta_{A, C} \Theta(B>C)
$$

For the fourth term, we have

$$
T_{4}=\sum_{I=1}^{n} \delta_{I, C+1} \sum_{J_{2}=1}^{B} \sum_{J_{3}=1}^{A} \delta_{J_{2}, I} \delta_{J_{3}, I}
$$




$$
\begin{aligned}
& =\sum_{J_{2}=1}^{B} \sum_{J_{3}=1}^{A} \delta_{J_{2}, C+1} \delta_{J_{3}, C+1} \\
& =\Theta(B>C) \Theta(A>C)
\end{aligned}
$$

Continuing, we have

$$
\begin{aligned}
T_{5} & =B A \sum_{I} \delta_{I, B+1} \delta_{I, A+1} \sum_{J_{1}=1}^{C} \delta_{J_{1}, I} \\
& =B A \delta_{B A} \sum_{I} \delta_{I, A+1} \sum_{J_{1}=1}^{C} \delta_{J_{1}, I} \\
& =B A \delta_{B, A} \Theta(A<C) \\
T_{6} & =-B \sum_{I} \delta_{I, B+1} \sum_{J_{1}=1}^{C} \sum_{J_{3}=1}^{A} \delta_{J_{3}, I} \delta_{J_{1}, I} \\
& =-\sum_{J_{1}=1}^{C} \sum_{J_{3}=1}^{A} \delta_{J_{3}, B+1} \delta_{J_{1}, B+1} \\
& =-B \Theta(B<C) \Theta(B<A) \\
T_{7} & =-A \sum_{I} \delta_{I, A+1} \sum_{J_{1}=1}^{C} \sum_{J_{2}=1}^{B} \delta_{J_{1}, I} \delta_{J_{2}, I} \\
& =-A \sum_{J_{1}=1}^{C} \sum_{J_{2}=1}^{B} \delta_{J_{1}, A+1} \delta_{J_{2}, A+1} \\
& =-A \Theta(A<C) \Theta(A<B) \\
T_{8} & =\sum_{I} \sum_{J_{1}=1}^{C} \sum_{J_{2}=1}^{B} \sum_{J_{3}=1}^{A} \delta_{J_{1}, I} \delta_{J_{2}, I} \delta_{J_{3}, I} \\
& =\sum_{J_{1}=1}^{B} \sum_{J_{2}=1}^{B} \sum_{J_{3}=1}^{A} \delta_{J_{1}, J_{3}} \delta_{J_{2}, J_{3}} \\
& =\sum_{J_{1}=1} \sum_{J_{2}=1}^{B} \sum_{J_{3}=1}^{A} \delta_{J_{1}, J_{2}, J_{3}} \\
& \operatorname{Min}(A, B, C) \\
& B(A) C
\end{aligned}
$$

Summing these terms gives (2.32).

\section{A.1 The $\kappa$ polynomial}

The 3-index invariant $\kappa_{A B C}$ can be used to define a symmetric polynomial in $z_{1}, z_{2}, \cdots, z_{n-1}$.

$$
\begin{aligned}
\kappa\left(z_{1}, z_{2}, \cdots, z_{n-1}\right) & =\sum_{A, B, C} \kappa_{A, B, C} z_{A} z_{B} z_{C} \\
= & -\sum_{A} A^{3} z_{A}^{3}+\sum_{A>B} B^{2} z_{A} z_{B}^{2}+\sum_{C>A} A^{2} z_{A}^{2} z_{C}+\sum_{A<B} A^{2} z_{A}^{2} z_{B}-\sum_{C<A ; C<B} C z_{A} z_{B} z_{C}
\end{aligned}
$$




$$
\begin{aligned}
& -\sum_{B<C ; B<A} B z_{A} z_{B} z_{C}-\sum_{A<B ; A<C} A z_{A} z_{B} z_{C}+\sum_{A, B, C} \operatorname{Min}(A, B, C) z_{A} z_{B} z_{C} \quad \text { (A.12) } \\
= & -\sum_{A} A^{3} z_{A}^{3}+3 \sum_{A<B} A^{2} z_{A}^{2} z_{B}-3 \sum_{A<B, A<C} A z_{A} z_{B} z_{C}+\sum_{A, B, C} \operatorname{Min}(A, B, C) z_{A} z_{B} z_{C}
\end{aligned}
$$

We used a renaming of summation variables to get the last line. The third term can be manipulated by separating into the case $B=C$, the case $A<B<C$ and the case $A<C<B$ to give

$$
\begin{aligned}
& -3 \sum_{A<B, A<C} A z_{A} z_{B} z_{C}=-3 \sum_{A<B} A z_{A} z_{B}^{2}-3 \sum_{A<B<C} A z_{A} z_{B} z_{C}-3 \sum_{A<C<B} A z_{A} z_{B} z_{C} \\
& \quad=-3 \sum_{A<B} A z_{A} z_{B}^{2}-6 \sum_{A<B<C} A z_{A} z_{B} z_{C}
\end{aligned}
$$

The last term can be separated into the cases $A=B=C$, the case where two are equal and smaller than the third, and the case where two are equal and larger than the third, and the case where all are different

$$
\sum_{A, B, C} \operatorname{Min}\left(z_{A}, z_{B}, z_{C}\right) z_{A} z_{B} z_{C}=\sum_{A} A z_{A}^{3}+3 \sum_{A<B} A z_{A}^{2} z_{B}+3 \sum_{A<B} A z_{A} z_{B}^{2}+6 \sum_{A<B<C} A z_{A} z_{B} z_{C}
$$

Collecting all the terms leads to some cancellations and simplifications:

$$
\kappa\left(z_{A}\right)=\sum_{A} A\left(1-A^{2}\right) z_{A}^{3}+\sum_{A<B} 3 A(1+A) z_{A}^{2} z_{B}
$$

In particular there are no terms where all the indices are different.

Consider now the polynomial $\kappa_{A}\left(z_{1}, z_{2}, \cdots, z_{n-1}\right)$

$$
\kappa_{A}(z)=\sum_{B, C} \kappa_{A B C} z_{B} z_{C}
$$

Consider

$$
\begin{aligned}
\frac{\partial \kappa(z)}{\partial z_{a}} & =\frac{\partial}{\partial z_{a}} \sum_{A, B, C} \kappa_{A B C} z_{A} z_{B} z_{C} \\
& =3 \sum_{B, C} \kappa_{a B C} z_{B} z_{C}=3 \kappa_{a}(z)
\end{aligned}
$$

So we find

$$
\kappa_{a}(z)=\frac{1}{3} \frac{\partial \kappa(z)}{\partial z_{a}}
$$

Now use the result (A.15) to find

$$
\kappa_{a}(z)=a\left(1-a^{2}\right) z_{a}^{2}+\sum_{A<a} A(1+A) z_{A}^{2}+\sum_{a<B} 2 a(1+a) z_{B} z_{a}
$$

Rewrite

$$
\kappa_{A}(z)=A\left(1-A^{2}\right) z_{A}^{2}+\sum_{B<A} B(1+B) z_{B}^{2}+\sum_{A<B} 2 A(1+A) z_{B} z_{A}
$$


Using the above polynomial, we find the explicit form of the constraints to be

For $1 \leq A \leq(n-1)$ :

$A\left(1-A^{2}\right) X_{\mu}^{(A)} X_{\mu}^{(A)}+\sum_{B: B>A} 2 A(1+A) X_{\mu}^{(A)} X_{\mu}^{(B)}+\sum_{B: B<A} B(1+B) X_{\mu}^{(B)} X_{\mu}^{(B)}=0$

and

$\sum_{A=1}^{n-1} X_{\mu}^{(A)} X_{\mu}^{(A)}=0$

\section{B Examples of $\mathcal{R} / \mathcal{I}$ at low $n, d$}

Recall that $V$ is the representation of $s o(4,2)$ that has all the states which correspond, by the operator-state correspondence, to the fundamental field and its derivatives. The unrefined generating function for the fundamental field of $s o(4,2)$ is (the factor in front of the trace below removes the contribution from the dimension of the scalar field itself)

$$
s^{\frac{2-d}{2}} \operatorname{tr}_{V}\left(s^{D}\right)=\frac{\left(1-s^{2}\right)}{(1-s)^{4}}=\frac{(1+s)}{(1-s)^{3}}
$$

This is exactly the unrefined Hilbert series for the conifold $\mathcal{C}$ (see eq. (4.5) of [28]. This is not an accident: the conifold is the solution set of

$$
z_{1}^{2}+z_{2}^{2}+z_{3}^{2}+z_{4}^{2}=0
$$

There are 4 generators and one quadratic relation, which matches the $s o(4,2)$ problem. In constructing the basic irrep for the free scalar field, we look at states constructed by acting with $P_{\mu}$ on the ground state, and set to zero the equation of motion $P_{\mu} P_{\mu}=0$.

For the case of the $d=4, n=3$ primaries (skipping the $n=2$ case where we have null vectors to deal with), we have the unrefined counting function

$$
\frac{\left(1-s^{2}\right)^{3}}{(1-s)^{8}}
$$

This answer is intuitive: as we have explained, here we are looking at polynomials in 3 coordinates $x_{\mu}^{1}, x_{\mu}^{2}, x_{\mu}^{3}$. After solving the $\mathrm{COM}$ condition we have polynomials in the two coordinates $X_{\mu}^{1}, X_{\mu}^{2}$ which are annihilated by the Laplacian in $X^{1}$, the Laplacian in $X^{2}$, and by

$$
\sum_{\mu=1}^{d} \frac{\partial^{2}}{\partial X_{\mu}^{(1)} \partial X_{\mu}^{(2)}}
$$

This ring should be the ring of polynomials on $\mathcal{C}^{2}$ subject to the condition

$$
\sum_{\mu=1}^{d} Z_{\mu}^{(1)} Z_{\mu}^{(2)}=0
$$


So the counting of states in this case corresponds to a subvariety in the product of two copies of the conifold.

In $d=3$ the ring with generators $z_{1}, z_{2}, z_{3}$ with relation

$$
z_{1}^{2}+z_{2}^{2}+z_{3}^{2}=0
$$

is the $A 1$ singularity. So the $n=3$ problem is a subvariety of the product of two $A 1$ singularity.

It is easy to make further connections between the $s o(3,2))$ counting of states in $V^{\otimes n}$ and the algebraic geometry of subvarieties. These connections can be verified using the SAGE computer package. For example, if $n=4$ we have the following four constraints (we have rescaled $X^{(3)}$ by $\sqrt{2}$ )

$$
\begin{aligned}
X^{(1)} \cdot X^{(2)}+X^{(1)} \cdot X^{\prime(3)} & =0 \\
X^{(1)} \cdot X^{(1)}-X^{(2)} X^{(2)}+X^{(2)} \cdot X^{\prime(3)} & =0 \\
X^{(1)} \cdot X^{(1)}+X^{(2)} \cdot X^{(2)}-4 X^{\prime(3)} \cdot X^{\prime(3)} & =0 \\
X^{(1)} \cdot X^{(1)}+X^{(2)} \cdot X^{(2)}+2 X^{\prime(3)} \cdot X^{\prime(3)} & =0
\end{aligned}
$$

Setting $d=3$ we have checked that we obtain the correct Hilbert series, by using the sage commands

R. $<x 1, x 2, x 3, y 1, y 2, y 3, z 1, z 2, z 3>=$ PolynomialRing (QQ, 9); R

I $=$ Ideal $([$

$\mathrm{x} 1 * \mathrm{y} 1+\mathrm{x} 2 * \mathrm{y} 2+\mathrm{x} 3 * \mathrm{y} 3+\mathrm{x} 1 * \mathrm{z} 1+\mathrm{x} 2 * \mathrm{z} 2+\mathrm{x} 3 * \mathrm{z} 3$,

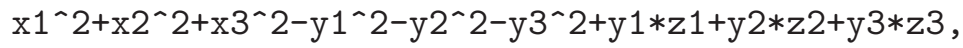

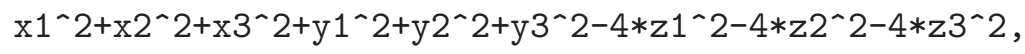

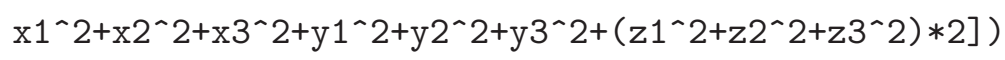

sage: I.hilbert_series()

The Hilbert series we obtain is

$$
H(s)=\frac{\left(1-s^{2}\right)^{4}}{(1-s)^{9}}
$$

which is indeed correct.

For $n=5$, after a suitable rescaling, we have the following five constraints:

$$
\begin{aligned}
X^{(1)} \cdot X^{(2)}+X^{(1)} \cdot X^{(3)}+X^{(1)} \cdot X^{(4)} & =0 \\
2 X^{(1)} \cdot X^{(1)}-6 X^{(2)} X^{(2)}+12 X^{(2)} \cdot X^{(3)}+12 X^{(2)} \cdot X^{(4)} & =0 \\
2 X^{(1)} \cdot X^{(1)}+6 X^{(2)} \cdot X^{(2)}-24 X^{(3)} \cdot X^{(3)}+24 X^{(4)} \cdot X^{(3)} & =0 \\
2 X^{(1)} \cdot X^{(1)}+6 X^{(2)} \cdot X^{(2)}+12 X^{(3)} \cdot X^{(3)}-60 X^{(4)} \cdot X^{(4)} & =0 \\
2 X^{(1)} \cdot X^{(1)}+6 X^{(2)} \cdot X^{(2)}+12 X^{(3)} \cdot X^{(3)}+20 X^{(4)} \cdot X^{(4)} & =0
\end{aligned}
$$

Using the sage commands

$\mathrm{R} .<\mathrm{x} 1, \mathrm{x} 2, \mathrm{x} 3, \mathrm{y} 1, \mathrm{y} 2, \mathrm{y} 3, \mathrm{z} 1, \mathrm{z} 2, \mathrm{z} 3, \mathrm{w} 1, \mathrm{w} 2, \mathrm{w} 3>=$ PolynomialRing $(\mathrm{QQ}, 12) ; \mathrm{R}$ I $=$ Ideal $([$

$\mathrm{x} 1 * \mathrm{y} 1+\mathrm{x} 2 * \mathrm{y} 2+\mathrm{x} 3 * \mathrm{y} 3+\mathrm{x} 1 * \mathrm{z} 1+\mathrm{x} 2 * \mathrm{z} 2+\mathrm{x} 3 * \mathrm{z} 3+\mathrm{x} 1 * \mathrm{w} 1+\mathrm{x} 2 * \mathrm{w} 2+\mathrm{x} 3 * \mathrm{w} 3$, 


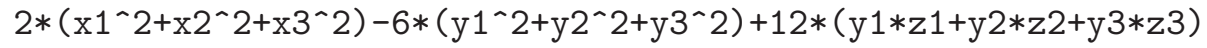

$+12 *(\mathrm{y} 1 * \mathrm{w} 1+\mathrm{y} 2 * \mathrm{w} 2+\mathrm{y} 3 * \mathrm{w} 3)$,

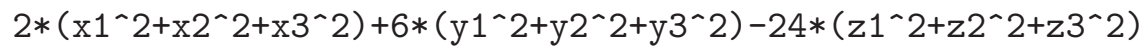

$+24 *(\mathrm{z} 1 * \mathrm{w} 1+\mathrm{z} 2 * \mathrm{w} 2+\mathrm{z} 3 * \mathrm{w} 3)$,

$2 *\left(\mathrm{x} 1^{\wedge} 2+\mathrm{x} 2 \wedge 2+\mathrm{x} 3^{\wedge} 2\right)+6 *\left(\mathrm{y} 1^{\wedge} 2+\mathrm{y} 2 \wedge 2+\mathrm{y} 3^{\wedge} 2\right)+12 *\left(\mathrm{z} 1^{\wedge} 2+\mathrm{z} 2^{\wedge} 2+\mathrm{z} 3^{\wedge} 2\right)$

$-60 *\left(\mathrm{w} 1 \wedge 2+\mathrm{w} 2 \wedge 2+\mathrm{w} 33^{\wedge} 2\right)$,

$2 *\left(\mathrm{x} 1^{\wedge} 2+\mathrm{x} 2 \wedge 2+\mathrm{x} 3^{\wedge} 2\right)+6 *\left(\mathrm{y} 1^{\wedge} 2+\mathrm{y} 2^{\wedge} 2+\mathrm{y} 3^{\wedge} 2\right)+12 *\left(\mathrm{z} 1^{\wedge} 2+\mathrm{z} 2^{\wedge} 2+\mathrm{z} 3^{\wedge} 2\right)$

$\left.\left.+20 *\left(w 1^{\wedge} 2+w 2 \wedge 2+w 3^{\wedge} 2\right)\right]\right)$

I.hilbert_series()

we again obtain the correct Hilbert series

$$
H(s)=\frac{\left(1-s^{2}\right)^{5}}{(1-s)^{12}}
$$

\section{Derivation of a symmetric group multiplicity formula for $V_{H}^{\otimes k}$}

The multiplicity of $S_{n} \times S_{k}$ irreps in $V_{H}^{\otimes k}$, where $V_{H}$ is the $S_{n}$ irrep associated with Young diagram $[n-1,1]$, is obtained by taking the trace of appropriate projectors which are expressible using characters [11]. We have (see for example [23] for further explanation of this formula)

$$
\begin{aligned}
\operatorname{Dim}\left(V_{\Lambda_{2}, \Lambda_{1}}\right) & =\operatorname{Mult}\left(V_{H}^{\otimes k}, V_{\Lambda_{1}}^{S_{n}} \otimes V_{\Lambda_{2}}^{S_{k}}\right) \\
& =\frac{1}{n ! k !} \sum_{\sigma \in S_{n}} \sum_{\tau \in S_{k}} \chi_{\Lambda_{1}}(\sigma) \chi_{\Lambda_{2}}(\tau) \prod_{i}\left(\operatorname{tr}_{H}\left(\sigma^{i}\right)\right)^{C_{i}(\tau)} \\
& =\sum_{p \vdash n} \sum_{q \vdash k} \frac{\chi_{\Lambda_{1}}^{p} \chi_{\Lambda_{2}}^{q}}{\operatorname{Symp} p \quad \text { Sym q }} \prod_{i}\left(\operatorname{tr}_{H}\left(\sigma_{p}^{i}\right)\right)^{q_{i}}
\end{aligned}
$$

Here $\sigma_{p}$ is a perm with cycle structure $p$. Now note that we have

$$
\begin{aligned}
\operatorname{tr}_{V_{H}}(\sigma) & =t r_{\text {nat }}(\sigma)-t r_{\text {triv }}(\sigma) \\
& =C_{1}(\sigma)-1
\end{aligned}
$$

and

$$
\begin{aligned}
\operatorname{tr}_{H}\left(\sigma^{i}\right) & =C_{1}\left(\sigma^{i}\right)-1 \\
& =-1+\sum_{d \mid i} d C_{d}(\sigma)
\end{aligned}
$$

When we raise a permutation to power $i$, all cycles of length $d$ which divide $i$ split into $d$ cycles of length 1 . It follows

$$
\operatorname{Dim}\left(V_{\Lambda_{2}, \Lambda_{1}}\right)=\sum_{p \vdash n} \sum_{q \vdash k} \frac{\chi_{\Lambda_{1}}^{p} \chi_{\Lambda_{2}}^{q}}{\operatorname{Sym} p \quad \operatorname{Symq}} \prod_{i=1}^{k}\left(-1+\sum_{d \mid i} d p_{d}\right)^{q_{i}}
$$




\section{Refined counting of LWPs in $d=3$ dimensions: tables}

In this appendix we summarize multiplicities for primaries constructed using $n$ fields and $k$ derivatives. These primaries transform in the spin $l$ representation of $s o(3)$ and in the $\Lambda_{n}$ of $S_{n}$.

\begin{tabular}{|l|c|c|}
\hline$l$ & $\Lambda_{3}$ & Mult \\
\hline 2 & {$[3]$} & 1 \\
\hline 2 & {$[2,1]$} & 1 \\
\hline 1 & {$[1,1,1]$} & 1 \\
\hline
\end{tabular}

Table 1. Results for $n=3$ fields and $k=2$ derivatives.

\begin{tabular}{|c|c|c|}
\hline$l$ & $\Lambda_{3}$ & Mult \\
\hline 3 & {$[3]$} & 1 \\
\hline 2 & {$[2,1]$} & 1 \\
\hline 3 & {$[2,1]$} & 1 \\
\hline 3 & {$[1,1,1]$} & 1 \\
\hline
\end{tabular}

Table 2. Results for $n=3$ fields and $k=3$ derivatives.

\begin{tabular}{|c|c|c|}
\hline$l$ & $\Lambda_{3}$ & Mult \\
\hline 4 & {$[3]$} & 1 \\
\hline 3 & {$[2,1]$} & 1 \\
\hline 4 & {$[2,1]$} & 2 \\
\hline 3 & {$[1,1,1]$} & 1 \\
\hline
\end{tabular}

Table 3. Results for $n=3$ fields and $k=4$ derivatives. 


\begin{tabular}{|c|c|c|}
\hline$l$ & $\Lambda_{4}$ & Mult \\
\hline 3 & {$[4]$} & 1 \\
\hline 1 & {$[3,1]$} & 1 \\
\hline 2 & {$[3,1]$} & 1 \\
\hline 3 & {$[3,1]$} & 2 \\
\hline 2 & {$[2,2]$} & 1 \\
\hline 1 & {$[2,1,1]$} & 1 \\
\hline 2 & {$[2,1,1]$} & 1 \\
\hline 3 & {$[2,1,1]$} & 1 \\
\hline 0 & {$[1,1,1,1]$} & 1 \\
\hline
\end{tabular}

Table 4. Results for $n=4$ fields and $k=3$ derivatives.

\begin{tabular}{|c|c|c|}
\hline$l$ & $\Lambda_{5}$ & Mult \\
\hline 3 & {$[5]$} & 1 \\
\hline 1 & {$[4,1]$} & 1 \\
\hline 2 & {$[4,1]$} & 1 \\
\hline 3 & {$[4,1]$} & 2 \\
\hline 1 & {$[3,2]$} & 1 \\
\hline 2 & {$[3,2]$} & 1 \\
\hline 3 & {$[3,2]$} & 1 \\
\hline 1 & {$[3,1,1]$} & 1 \\
\hline 2 & {$[3,1,1]$} & 1 \\
\hline 3 & {$[3,1,1]$} & 1 \\
\hline 1 & {$[2,2,1]$} & 1 \\
\hline 2 & {$[2,2,1]$} & 1 \\
\hline 0 & {$[2,1,1,1]$} & 1 \\
\hline
\end{tabular}

Table 5. Results for $n=5$ fields and $k=3$ derivatives. 


\section{E Refined counting of LWPs in $d=4$ dimensions: tables}

We give the multiplicities for primaries constructed using $n$ fields and $k$ derivatives. These primaries transform in the spin $\left(l_{1}, l_{2}\right)$ representation of $s o(4)$ and in the $\Lambda_{n}$ of $S_{n}$.

\begin{tabular}{|c|c|c|c|}
\hline$l_{1}$ & $l_{2}$ & $\Lambda_{3}$ & Mult \\
\hline 1 & 1 & {$[3]$} & 1 \\
\hline 1 & 1 & {$[2,1]$} & 1 \\
\hline 0 & 1 & {$[1,1,1]$} & 1 \\
\hline 1 & 0 & {$[1,1,1]$} & 1 \\
\hline
\end{tabular}

Table 6. Results for $n=3$ fields and $k=2$ derivatives.

\begin{tabular}{|c|c|c|c|}
\hline$l_{1}$ & $l_{2}$ & $\Lambda_{3}$ & Mult \\
\hline $3 / 2$ & $3 / 2$ & {$[3]$} & 1 \\
\hline $1 / 2$ & $3 / 2$ & {$[2,1]$} & 1 \\
\hline $3 / 2$ & $1 / 2$ & {$[2,1]$} & 1 \\
\hline $3 / 2$ & $3 / 2$ & {$[2,1]$} & 1 \\
\hline $3 / 2$ & $3 / 2$ & {$[1,1,1]$} & 1 \\
\hline
\end{tabular}

Table 7. Results for $n=3$ fields and $k=3$ derivatives.

\begin{tabular}{|c|c|c|c|}
\hline$l_{1}$ & $l_{2}$ & $\Lambda_{3}$ & Mult \\
\hline 0 & 2 & {$[3]$} & 1 \\
\hline 2 & 0 & {$[3]$} & 1 \\
\hline 2 & 2 & {$[3]$} & 1 \\
\hline 1 & 2 & {$[2,1]$} & 1 \\
\hline 2 & 1 & {$[2,1]$} & 1 \\
\hline 2 & 2 & {$[2,1]$} & 2 \\
\hline 1 & 2 & {$[1,1,1]$} & 1 \\
\hline 2 & 1 & {$[1,1,1]$} & 1 \\
\hline
\end{tabular}

Table 8. Results for $n=3$ fields and $k=4$ derivatives. 


\begin{tabular}{|c|c|c|c|}
\hline$l_{1}$ & $l_{2}$ & $\Lambda_{4}$ & Mult \\
\hline $3 / 2$ & $3 / 2$ & {$[4]$} & 1 \\
\hline $1 / 2$ & $1 / 2$ & {$[3,1]$} & 1 \\
\hline $1 / 2$ & $3 / 2$ & {$[3,1]$} & 1 \\
\hline $3 / 2$ & $1 / 2$ & {$[3,1]$} & 1 \\
\hline $3 / 2$ & $3 / 2$ & {$[3,1]$} & 2 \\
\hline $1 / 2$ & $3 / 2$ & {$[2,2]$} & 1 \\
\hline $3 / 2$ & $1 / 2$ & {$[2,2]$} & 1 \\
\hline $1 / 2$ & $1 / 2$ & {$[2,1,1]$} & 1 \\
\hline $1 / 2$ & $3 / 2$ & {$[2,1,1]$} & 1 \\
\hline $3 / 2$ & $1 / 2$ & {$[2,1,1]$} & 1 \\
\hline $3 / 2$ & $3 / 2$ & {$[2,1,1]$} & 1 \\
\hline $1 / 2$ & $1 / 2$ & {$[1,1,1,1]$} & 1 \\
\hline
\end{tabular}

Table 9. Results for $n=4$ fields and $k=3$ derivatives.

\begin{tabular}{|c|c|c|c|}
\hline$l_{1}$ & $l_{2}$ & $\Lambda_{5}$ & Mult \\
\hline $3 / 2$ & $3 / 2$ & {$[5]$} & 1 \\
\hline $1 / 2$ & $1 / 2$ & {$[4,1]$} & 1 \\
\hline $1 / 2$ & $3 / 2$ & {$[4,1]$} & 1 \\
\hline $3 / 2$ & $1 / 2$ & {$[4,1]$} & 1 \\
\hline $3 / 2$ & $3 / 2$ & {$[4,1]$} & 2 \\
\hline $1 / 2$ & $1 / 2$ & {$[3,2]$} & 1 \\
\hline $1 / 2$ & $3 / 2$ & {$[3,2]$} & 1 \\
\hline $3 / 2$ & $1 / 2$ & {$[3,2]$} & 1 \\
\hline $3 / 2$ & $3 / 2$ & {$[3,2]$} & 1 \\
\hline $1 / 2$ & $1 / 2$ & {$[3,1,1]$} & 1 \\
\hline $1 / 2$ & $3 / 2$ & {$[3,1,1]$} & 1 \\
\hline $3 / 2$ & $1 / 2$ & {$[3,1,1]$} & 1 \\
\hline $3 / 2$ & $3 / 2$ & {$[3,1,1]$} & 1 \\
\hline $1 / 2$ & $1 / 2$ & {$[2,2,1]$} & 1 \\
\hline $1 / 2$ & $3 / 2$ & {$[2,2,1]$} & 1 \\
\hline $3 / 2$ & $1 / 2$ & {$[2,2,1]$} & 1 \\
\hline $1 / 2$ & $1 / 2$ & {$[2,1,1,1]$} & 1 \\
\hline
\end{tabular}

Table 10. Results for $n=5$ fields and $k=3$ derivatives. 
Open Access. This article is distributed under the terms of the Creative Commons Attribution License (CC-BY 4.0), which permits any use, distribution and reproduction in any medium, provided the original author(s) and source are credited.

\section{References}

[1] R. de Mello Koch, P. Rabambi, R. Rabe and S. Ramgoolam, Free quantum fields in $4 D$ and Calabi-Yau spaces, Phys. Rev. Lett. 119 (2017) 161602 [arXiv:1705. 04039] [INSPIRE].

[2] R. de Mello Koch, P. Rabambi, R. Rabe and S. Ramgoolam, Counting and construction of holomorphic primary fields in free $\mathrm{CFT}_{4}$ from rings of functions on Calabi-Yau orbifolds, JHEP 08 (2017) 077 [arXiv: 1705.06702] [INSPIRE].

[3] B. Henning, X. Lu, T. Melia and H. Murayama, Operator bases, S-matrices and their partition functions, JHEP 10 (2017) 199 [arXiv:1706.08520] [INSPIRE].

[4] R. de Mello Koch and S. Ramgoolam, $C_{F T}$ as $\mathrm{SO}(4,2)$-invariant $T F T_{2}$, Nucl. Phys. B 890 (2014) 302 [arXiv:1403.6646] [INSPIRE].

[5] F.A. Dolan, Character formulae and partition functions in higher dimensional conformal field theory, J. Math. Phys. 47 (2006) 062303 [hep-th/0508031] [INSPIRE].

[6] D.A. Cox, J.B. Little and D. O'Shea, Ideals, varieties, and algorithms, fourth edition, Springer, Cham, Switzerland, (2015).

[7] D. Eisenbud, Commutative algebra. With a view toward algebraic geometry, Grad. Texts Math. 150, Springer-Verlag, New York, U.S.A., (1995) [ISBN:0-387-94268-8].

[8] E. Grigorescu, Hilbert series and free resolutions, Senior thesis, Bard College, Annandale-on-Hudson, NY, U.S.A., (2003).

[9] G. Wu, Koszul algebras and Koszul duality, Masters thesis, University of Ottawa, Ottawa, ON, Canada, (2016).

[10] R.C. King, Young tableaux, Schur functions and $\mathrm{SU}(2)$ plethysms, J. Phys. A 18 (1985) 2429.

[11] W. Fulton and J. Harris, Representation theory: a first course, Springer, New York, U.S.A., (1991).

[12] C.W. Ayoub, On constructing bases for ideals in polynomial rings over the integers, J. Num. Theor. 17 (1983) 204.

[13] R. De Mello Koch, P. Rambambi and H.J.R. Van Zyl, From spinning primaries to permutation orbifolds, JHEP 04 (2018) 104 [arXiv: 1801.10313] [INSPIRE].

[14] A. Ram, Characters of Brauer's centralizer algebras, Pacific J. Math. 169 (1985) 173.

[15] S. Corley, A. Jevicki and S. Ramgoolam, Exact correlators of giant gravitons from dual $N=4$ SYM theory, Adv. Theor. Math. Phys. 5 (2002) 809 [hep-th/0111222] [INSPIRE].

[16] J. Pasukonis and S. Ramgoolam, Quivers as calculators: counting, correlators and Riemann surfaces, JHEP 04 (2013) 094 [arXiv:1301.1980] [INSPIRE].

[17] Y. Kimura, Noncommutative Frobenius algebras and open-closed duality, arXiv:1701.08382 [INSPIRE].

[18] P. Mattioli and S. Ramgoolam, Permutation centralizer algebras and multi-matrix invariants, Phys. Rev. D 93 (2016) 065040 [arXiv:1601.06086] [INSPIRE]. 
[19] R. Bhattacharyya, S. Collins and R. de Mello Koch, Exact multi-matrix correlators, JHEP 03 (2008) 044 [arXiv:0801.2061] [INSPIRE].

[20] R. Bhattacharyya, R. de Mello Koch and M. Stephanou, Exact multi-restricted Schur polynomial correlators, JHEP 06 (2008) 101 [arXiv:0805.3025] [INSPIRE].

[21] Y. Kimura and S. Ramgoolam, Branes, anti-branes and Brauer algebras in gauge-gravity duality, JHEP 11 (2007) 078 [arXiv:0709.2158] [INSPIRE].

[22] T.W. Brown, P.J. Heslop and S. Ramgoolam, Diagonal multi-matrix correlators and BPS operators in $N=4$ SYM, JHEP 02 (2008) 030 [arXiv:0711.0176] [INSPIRE].

[23] T.W. Brown, P.J. Heslop and S. Ramgoolam, Diagonal free field matrix correlators, global symmetries and giant gravitons, JHEP 04 (2009) 089 [arXiv:0806.1911] [INSPIRE].

[24] A. Polischchuk and L. Positselski, Quadratic algebras, Univ. Lect. Ser. 37, American Mathematical Society, Providence, RI, U.S.A., (2005).

[25] Koszul duality Wikipedia article, https://en.wikipedia.org/wiki/Koszul_duality.

[26] R.J. Szabo, Quantum field theory on noncommutative spaces, Phys. Rept. 378 (2003) 207 [hep-th/0109162] [INSPIRE].

[27] R. Fröberg and C. Löfwal, Koszul homology and Lie algebras with application to generic forms and points, Homology Homotopy Appl. 4 (2002) 227.

[28] S. Benvenuti, B. Feng, A. Hanany and Y.-H. He, Counting BPS operators in gauge theories: quivers, Syzygies and Plethystics, JHEP 11 (2007) 050 [hep-th/0608050] [INSPIRE]. 